\title{
IMPACT OF INDOOR LOCATION INFORMATION RELIABILITY ON USERS' TRUST OF AN INDOOR POSITIONING SYSTEM
}

\author{
A Thesis Submitted to the College of \\ Graduate Studies and Research \\ In Partial Fulfillment of the Requirements \\ For the Degree of Master of Science \\ In the Department of Geography and Planning \\ University of Saskatchewan \\ Saskatoon
}

By

Ting Wei

(c) Copyright Ting Wei, October, 2012. All rights reserved. 


\section{PERMISSION TO USE}

In presenting this thesis in partial fulfilment of the requirements for a Postgraduate degree from the University of Saskatchewan, I agree that the Libraries of this University may make it freely available for inspection. I further agree that permission for copying of this thesis in any manner, in whole or in part, for scholarly purposes may be granted by the professor or professors who supervised my thesis work or, in their absence, by the Head of the Department or the Dean of the College in which my thesis work was done. It is understood that any copying or publication or use of this thesis or parts thereof for financial gain shall not be allowed without my written permission. It is also understood that due recognition shall be given to me and to the University of Saskatchewan in any scholarly use which may be made of any material in my thesis.

Requests for permission to copy or to make other use of material in this thesis in whole or part should be addressed to:

Head of the Department of Geography and Planning

University of Saskatchewan

Saskatoon, Saskatchewan (S7N 5C8) 


\begin{abstract}
Indoor positioning systems have been used as a supplement to provide positioning in settings where GPS does not function. However, the accuracy of calculated results varies among techniques and algorithms used; system performance also differs across testing environments. As a result, users' responses to and opinions of these positioning results could be different. Furthermore, user trust, most closely associated with their confidence in the system, will also vary. A relatively little studied topic is the effect of positioning variance on a user's opinion or trust of such systems (GPS as well, for that matter). Therefore, understanding how user interaction with such systems (through trust) changes is important for achieving more usable positioning system design. An experiment was designed to examine if the sequence of location accuracy will affect users' trust in an individual episode positioning result as well as the system overall. The simulated positioning system running on an iPad used for this experiment provides 10 priming positioning results at a specific category of accuracy. The accuracy is controlled and is presented as either 1. ACCURATE (within 5 meters of actual location), 2. INACCURATE (greater 15 meters), or 3. WRONG BUILDING (outside current building's footprint). After one set of these priming locations a series of 55 post-priming locations across the same categories in addition to 10 CONTINUOUS locations (with between 6 and 15 meters of error) were presented. At each experimental site participants located themselves using the simulated system and rated their trust for that location. Variables obtained from the experiment include: 1 . Two types of trust at each location (positioning trust and system trust); 2. Spatial abilities, sense of direction, and ancillary survey data (user characteristics). Results show that users' trust varies
\end{abstract}


among different accuracy categories and changes over time according to the system performance in association with their own characteristics. Specifically, the accuracy of the priming locations has an impact on users' trust of later results. Besides, users' trust in individual positioning results is quite variable and the variability is closely related to accuracy, while user trust of the overall system is less variable. 


\section{ACKNOWLEDGMENTS}

First of all, I would like to record my gratitude to my supervisor, Dr. Scott Bell, for his patient guidance, great advice from the very beginning of this research as well as the encouragement and support throughout the work. Without his contribution of time and ideas, this thesis would not have been possible. Meanwhile, I gratefully acknowledge my advisory committee Drs. Regan Mandryk, Cherie Westbrook, Paul Hackett as well as the external examiner Dr. Carl Gutwin for their direction and advice along this research.

I also want to extend my special thanks to my department faculties, staffs and fellow students for their tremendous help during my study, research and everyday life. It is also impossible to overvalue the contribution of family and friends, their constant love and support has enabled me to complete my master study.

Finally, I appreciate the financial support from the Canadian National Centers for Excellence, GEOmatics for Informed DEcisions (GEOIDE) and Social Sciences and Humanities Research Council (SSHRC). I also want to thank University of Saskatchewan Facilities Management Division for their willingness to share the campus map data. In addition, the Spatial Analysis for Innovation in Health Research (SAFIHR) Lab with funding from Canadian Foundation for Innovation (CFI) has provided space and equipment I have needed to produce and complete this research. 


\section{CONTENTS}

I INTRODUCTION ...................................................................................... 1

1.1 Background ....................................................................................... 1

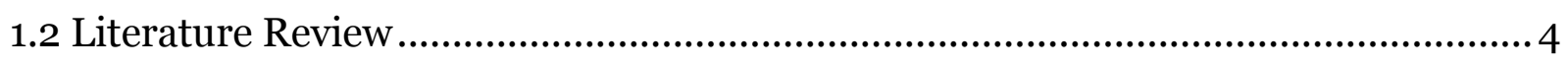

1.2.1 Positioning Accuracy ...................................................................... 5

1.2.1.1 System Accuracy ....................................................................... 5

1.2.1.2 System Reliability ..................................................................... 6

1.2.2 Interacting with a Positioning System ................................................. 7

1.2.2.1 Context Awareness .......................................................................... 7

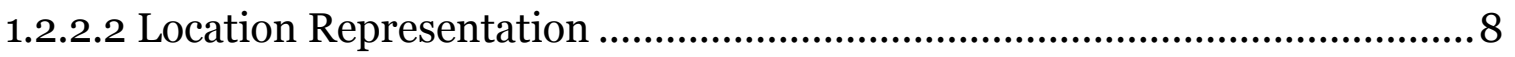

1.2.2.3 Handling Location Uncertainty ....................................................... 10

1.2.3 User Opinion of Location-based Systems ...............................................11

1.2.3.1 System Usability Evaluation ....................................................... 11

1.2.3.2 System Accuracy Evaluation ......................................................... 12

1.2.4 User Trust of Location-based Systems .................................................. 13

1.2.4.1 Definition and Impact of Trust ....................................................... 13

1.2.4.2 Dynamic Nature of Trust Change....................................................... 14

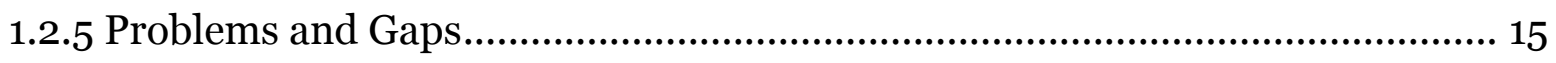

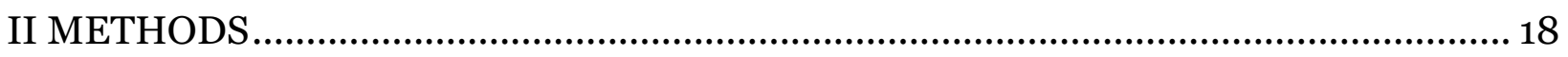

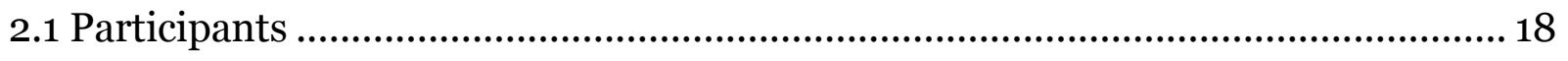

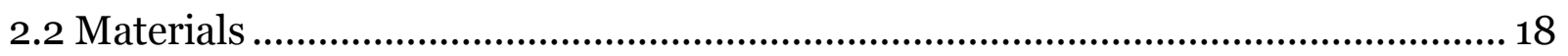

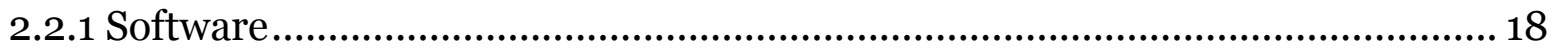

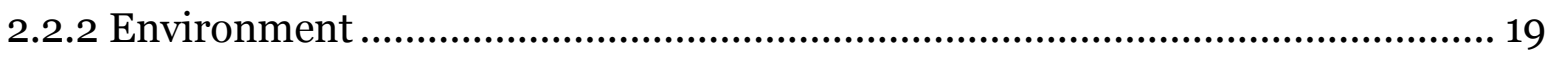

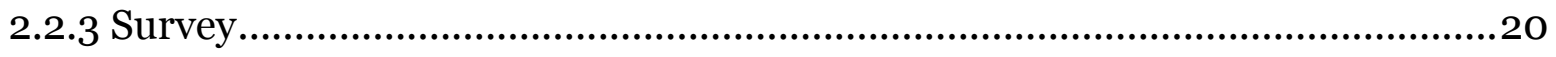

2.2.4 Spatial Ability Tasks ....................................................................20

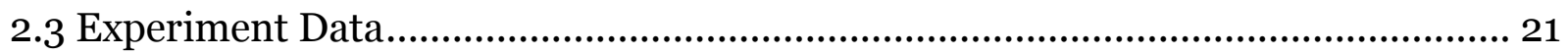

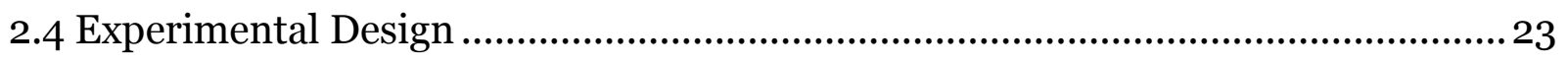

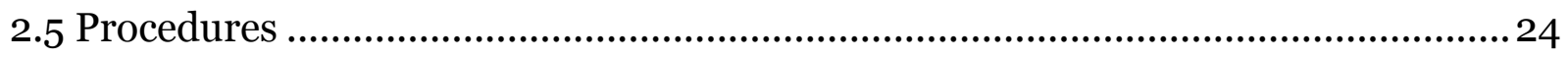

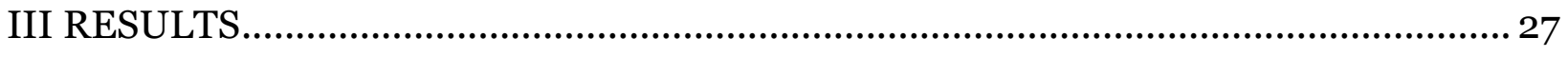




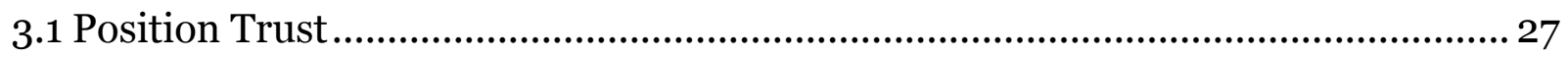

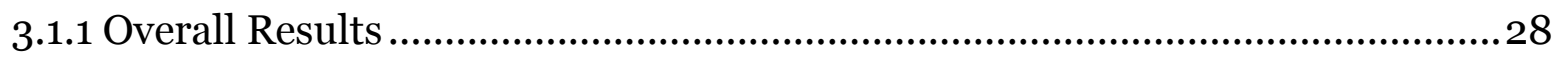

3.1.2 Priming Effect on Position Trust ........................................................... 29

3.1.3 Position Trust of Locations in the Same Category ....................................... 32

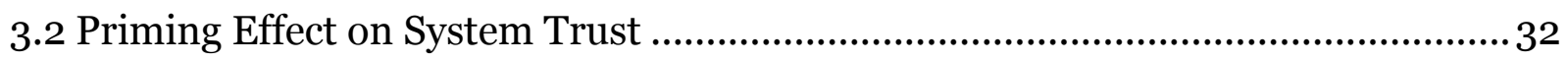

3.3 Comparison between Position Trust and System Trust .......................................33

3.4 Effect of Positioning Error on Position Trust ............................................... 36

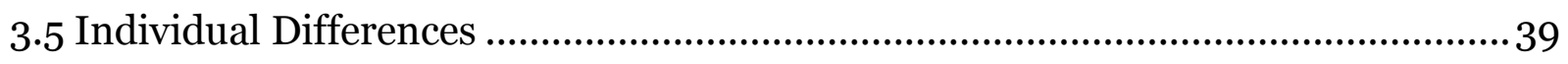

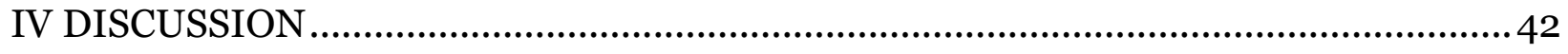

4.1 Positioning Accuracy VS. Trust .............................................................. 42

4.2 Priming Effect on Trust ...................................................................... 43

4.3 Position Trust VS. System Trust ................................................................ 45

4.4 Individual Differences ......................................................................4 46

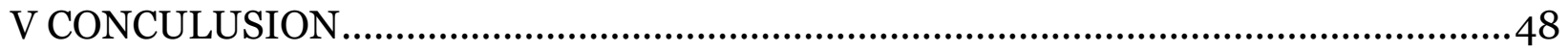

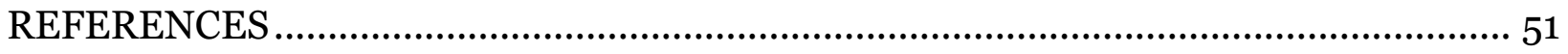

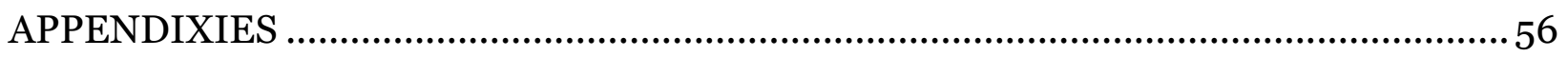




\section{TABLES}

Table 1. Four types of locations used in the priming and post-priming stage in three buildings ("K" represents Kirk Hall, "A" represents the Agriculture Building, and "E" represents the Engineering Building) ............................................ 22

Table 2. Building visiting sequences for each group 23

Table 3. Descriptive statistics for Position Trust (18 participants in each group, which includes 15 ACCURATE, 15 INACCURATE, and 15 WRONG BUILDING locations after priming locations) 28

Table 4. Calculated $p$ values from the Mann-Whitney U Test 31

Table 5. Significant scenarios of pair comparisons for position trust of three types of locations across groups

Table 6. Descriptive statistics for position trust of ACCURATE, INACCURAE and WRONG BUILDING locations in both priming stage and post-priming stage 32

Table 7. Descriptive statistics for system trust in post-priming stage 33

Table 8. Descriptive statistics for position trust (PT) and system trust (ST) at ACCUATE (T_A), INACCURATE (T_IA), and WRONG BUILDING (T_WB) locations in the post-priming stage 34

Table 9. $\mathrm{R}$ square values of different linear models describing relationship between positioning error and average position trust ratings of ACCURATE, CONTINUOUS and INACCURATE locations 37

Table 10. Descriptive statistics of data collected from spatial ability tests and surveys by gender (MRT: Mental Rotation Task score; OLMT: Object Location Memory Test score; AVG_SBSOD: average score of SBSOD; SUM_Familiarity: sum ratings of familiarity with all three buildings; GPS_Experience: rating of GPS experience; GPS_Principle: rating of understanding of the GPS principle) 


\section{FIGURES}

Figure 1. Data recording interface of the simulated SaskEPS ................................... 25

Figure 2. Mean values of position trust of three location types (PT_A: position trust of ACCURATE locations, PT_IA: position trust of INACCURATE locations; PT_WB: position trust of WRONG BUILDING locations; Groups, G1: ACCURATE priming, G2: INACCURATE priming, G3: WRONG BUILDING priming)

Figure 3. Mean values of position trust (PT) and system trust (ST) of three location types (A: trust of ACCURATE locations, IA: trust of INACCURATE locations; WB: trust of WRONG BUILDING locations)

Figure 4. Relationship between positioning error and average position trust ratings of ACCURATE, CONTINUOUS and INACCURATE locations (40 points included, positioning error $<=50 \mathrm{~m}$ )

Figure 5. Actual (where participants stand) and result location (calculated by the system) of points 31 (top) and 53 (bottom) (black star represents the actual location and black dot represents the result location). 


\section{I}

\section{INTRODUCTION}

\subsection{Background}

The Global Positioning System (GPS) has rapidly become the primary source for accurate location information anywhere in the world and under any weather conditions. Having reliable and accurate location information significantly helps in emergency services, navigation, commercial services, recreation, tracking, and networking (Zandbergen, 2009). Also, most cell phones include a GPS location chip for processing the GPS signal; these kinds of cell phone-based GPS systems are generally called Assisted GPS (A-GPS, GPS supplemented by cellular and WiFi and generally less accurate than GPS). The ability of mobile phones to determine their location in realtime can greatly help in urban search and rescue missions and point-to-point navigation (Zàruba, Huber, Kamangar, \& Chlamtac, 2007).

However, both GPS and A-GPS cannot provide reliable positioning results in indoor environments because of the relatively weak GPS signal's inability to penetrate building materials. As a result, several supplementary techniques have been used (Bluetooth, Cellular, wireless internet (WiFi), Radio Frequency ID (RFID), Ultra Wide Band (UWB), etc.) to provide positioning in indoor settings where GPS does not function (Georgy, Noureldin, Korenberg, \& Bayoumi, 2010a, 2010b). To obtain a device's current location, a positioning system needs to obtain contextual information from a user; a GPS might need to know whether a user is walking or driving, and the speed of the two transportation modes. Similarly, for an indoor positioning system, the handheld device cannot "read" a user's current location directly; the location information needs to be derived from alternative measurements obtained from the users' surroundings, such as 
received wireless signals and the various and specific signal strength value, angle, time, and signal strength distribution, depending on the different techniques used. Moreover, calculation is not the only job that a positioning system needs to do, users need to interact with the positioning system to achieve their specific navigation goal. For example, users need to initiate the device at the beginning of a route or a specific location and they need to interpret the results represented through the system interface. Also, positioning systems need to manage uncertainty (predict, communicate, etc.), as positioning results are not always accurate; sometimes they are not correct at all.

Under uncertain conditions a user's trust in positioning results may vary in conjunction with accuracy or might vary in a more complicated way related to their personal knowledge, experience, or the pattern of results they have experienced while using the system. However, inaccuracies caused by inadequate and inaccurate positioning source data, deficient techniques or algorithms, or an unclear GUI make it difficult to control or predict accuracy in real world settings. To make things more complicated, users appear to use perceived accuracy but not actual accuracy when judging system credibility according to the experimental results. Users likely rely on their cognitive map, which contains their spatial knowledge of the local surroundings, to locate themselves in the environment and compare that to what they see from a positioning system before making a trust assessment; however, the speed and accuracy of this process is highly dependent on a user's local knowledge, spatial ability, confidence, etc. As a result, it is important to understand how user trust changes with system performance as well as develop models of trust for systems to predict when results might be more uncertain. 
An experiment was designed to evaluate the impact of varying accuracy and reliability in indoor positioning on user trust. In particular, I was interested in how consistent positioning results in a category of accuracy (accurate, inaccurate, and nominally incorrect) would affect user trust of later "random" positioning results (random order of locations falling in different accuracy). The simulated positioning system (embedded in the Saskatchewan EXPerimental Design APP (SaskEXP) and installed on an iPad) used for this experiment presented 10 priming trials (each trial consisted of a single simulated positioning result) at a specific level of accuracy (ACCURATE, INACCURATE, and WRONG BUILDING, see methods section for details) before a random series of positioning results from the same categories in addition to 10 CONTINUOUS locations (with positioning error between 6 and 15 meters of error). Participants were given an iPad running the simulated positioning system that included the presentation of positioning results at pre-selected sites in the experimental area. At each point, participants were asked to rate their confidence in the positioning results and the positioning system. My expectation (hypothesis) was that starting with accurate results would cause higher trust ratings for inaccurate results presented later in the experiment. This hypothesis is based on the premise that a set of consistently accurate results presented in series would communicate to the user that the system is reliable to the extent that the user would "overlook" (have less distrust for) inaccurate positioning results presented in a following series that included results across a range of accuracies. On the contrary, starting (priming) with inaccurate results would negatively affect trust; users would continue using the system but would rate all subsequent results less trustworthy. Therefore, trust will be lower if initial location information is deemed untrustworthy. Furthermore, it was anticipated that system trust will be less variable 
across different types of locations. This hypothesis is based on the fact that the "system" is composed of the interface, base map data, and positioning results. As only one of three primary components, the variability of the positioning results will affect system trust less.

\subsection{Literature Review}

While many users of contemporary GPS accept as standard the highly accurate and reliable positioning information they are given (or that is used to deliver location based services (LBS)), positioning information from non-GPS sources is far from reliable or ubiquitously accurate. Take indoor positioning systems as an example, many positioning results are not accurate; on occasion it is even completely incorrect (outside a building when the user is in side, kilometres away, etc.). Such variability in system performance could have a substantial impact on a user's response and future use of such information. In addition to issues of accuracy, other problems involve location representation, system usability, and individual differences in interpreting accuracy. To attempt to solve these ongoing problems, a new topic receiving attention is the influence of the reliability and accuracy of positioning results on users' opinions of the system. Studies on this topic have raised the issue of trust as it relates to users' confidence in the positioning results. As inaccuracy can hardly be predicted, in order to maintain trust when systems are deployed, it is important to adjust the system appropriately during

periods of unexpected inaccuracy. Such adjustments require a better understanding of how users' trust changes with system performance over time. 


\subsubsection{Positioning Accuracy}

\subsubsection{System Accuracy}

The accuracy of positioning systems can vary based on the technique and algorithm used. When choosing and implementing those techniques and algorithms, the area of coverage, implementation cost, and calculation accuracy must be balanced. For most positioning methods, an increase in accuracy is associated with additional processing power, required equipment, and/or increased system latency (Beal, 2003). Several review articles have discussed the characteristics of each technique and algorithm: some techniques involve different signal systems (for example, cellular, Bluetooth, Radio Frequency ID (RFID), etc.). Each signal system has limitations: Bluetooth has a relatively short range; the accuracy of cellular systems is generally low; and additional hardware needs to be deployed when using RFID and the ultra wide band (UWB) technique (Liu, Darabi, Banerjee, \& Liu, 2007). The most common indoor positioning systems rely on either wireless Internet signals from routers (WiFi) or RFID. While different algorithms use those signals to calculate position in different ways (triangulation, trilateration, and fingerprinting), each of them have strengths, weaknesses and unique variables that affect accuracy (Gezici, 2008; Hazas, Scott, \& Krumm, 2004). The trilateration algorithm calibrates signal strength in real time and then uses the value as an approximation for distance to trilaterate a device's location; generally this technique is flexible and has a relatively low start-up cost (time and money). The fingerprinting technique relies on a comparison between the received signal strength (RSS) values from "visible" routers at a location and the signal strength values stored in a fingerprint database. Generally, fingerprinting requires time and effort to establish the database and it is sensitive to changes in router location. 
Triangulation uses geometric calculations to estimate the target location (Gezici, 2008). The TOA (Time of Arrival) or the AOA (Angle of Arrival) approach could be used for triangulation instead of measuring signal strength directly (as in trilateration), but additional devices are needed for measurements.

\subsubsection{System Reliability}

When evaluating the overall system performance, both accuracy and reliability should be considered. Accuracy is the localization error distance (the distance between the actual and calculated position) while reliability specifies how often the system attains that accuracy (range of accuracy across multiple locations) (Hightower \& Borriello, 2001). As positioning is a continuous real world process, the claimed accuracy is not always consistent with the actual accuracy at any given time and location. For example, the performance of Consumer-Grade GPS Receivers depends on canopy cover and satellite availability, which results in a variance in accuracy when used in different settings (Wing, Eklund, \& Kellogg, 2005). Skyhook's hybrid positioning system (XPS), which combines GPS, WiFi, and cellular signals, claims that its "core engine" has an accuracy of 10 meters and compares this accuracy level with GPS and A-GPS at 10 and 30 meters, respectively. However, in real world testing, the system fails to meet this level of accuracy (Bell, Jung, \& Krishnakumar, 2010; Zandbergen, 2009). The average error across hundreds of randomly chosen locations is over 60 meters, and for many locations, the estimated location is outside the footprint of the target building (Bell, et al., 2010). When these systems fail to achieve claimed accuracy users are

understandably frustrated. Similarly, indoor positioning systems face the challenge of maintaining consistent and continuous accuracy over both time and space. As signal propagation and attenuation are extremely complicated in indoor environments, these 
disturbances lead directly to highly variable results. In addition, accurate system calculation relies on the quality and completeness of the positioning source data (Bell, Wei, Jung, \& Scott, 2011). However, it is unrealistic for all buildings to have the same density of sensors, structure, or router arrangement, all of which contribute to variation in indoor positioning systems' reliability.

\subsubsection{Interacting with a Positioning System}

\subsubsection{Context Awareness}

The importance of context awareness in computing systems, including positioning systems and handheld devices cannot be understated (Wei \& Chan, 2007). Context is information that characterizes the situation of an entity or occurrence. Dey (2001) defines entity as "a person, place, or object that is considered relevant to the interaction between a user and an application, including the user and applications themselves." Context awareness in positioning system is the ability to be aware of a user's current location and to adjust the location according to environmental change (Gorlenko \& Merrick, 2003). For example, a GPS should know whether a user is walking or driving, and an indoor positioning system should be aware of the building in which the user is walking, as well as the user's current location. Location information is an important source of context and a core component of the user's interaction with the system, which needs to be accurate and reliable from the perspective of a user. In addition, context contributes significantly to a user's response, opinions, observable behavior, and judgments regarding the provided positioning information. As a result, users need to fully understand the context provided by the system. Such context information can facilitate real world use. However, the contextual information might be unclear to the user, an additional decision making hurdle for users. For example, when using Google 
Maps on the iPhone, one's position is indicated by a bright blue dot with a pulsating blue location circle around it. The state of the blue dot indicates positioning error; however, "positioning error" in this instance is only based on what technique is being used for determining position (a different style dot for cellular only, WiFi only, and for GPS) (Zandbergen, 2009). This design might not be apparent to naïve users, as these users have limited knowledge of the basic principles of positioning systems. They are unlikely to be sensitive to such subtle changes in the location display or understand the implications of these changes (Bell, et al., 2011).

\subsubsection{Location Representation}

A variety of information is provided to users when displaying positioning results, which include not only a user's current location but also basemap data. Different levels of location information are displayed to help users understand their current location: $\mathrm{XY}$ coordinates provide the precise location of positions in indoor spaces. However, uncertainty can be added if the result location coordinates are not accurate. For instance, when using AOA or TOA based technique, if a device can only detect signals from two Access Points (APs), the user's location will be represented somewhere between the two APs. Similarly, when using fingerprinting, the most probable user's location will be represented by that fingerprint's location in the database because location is determined by comparing received signal strength value with the fingerprint value in the database. These estimated locations sometimes prove to be points on the map where a user cannot possibly stand. In addition, users build local context through their visual assessment of the surrounding area (for example halls, rooms, etc.) (Beal, 2003), the detail of this information may affect the user's understanding of the

presented location. Researchers have made efforts to represent accurate 2D line 
basemaps of indoor environments using the Region and Gateway (RG) map, a laserbased 2D line mapping technique (Schröter, Beetz, \& Gutmann, 2002). However, this technique has only been tested by a small group of users and still needs to be deployed in a wider area, as well as thoroughly tested.

In addition, indoor spaces are generally considered qualitatively different from outdoor spaces ( $\mathrm{Li}, 2008)$. Structural elements constrain navigation freedom, limit available decisions, and reduce visual extent. Users can only move in a restricted area (hallways and rooms) in indoor environments (Shoeb, Ahmad, \& Amin, 2006). These constraints raise another concern for indoor positioning systems, which involves the conversion of positioning results (specific and precise coordinates) onto a map representation of actual places (Hightower, 2003), which is often a more nuanced geographic concept associated with the local surroundings. This means that an accurate positioning result that is only 1 metre from the correct location, but is placed on the wrong side of a building's wall, will be deemed as untrustworthy and quite inaccurate. Research has been done on converting absolute locations to relative locations (Hightower \& Borriello, 2001). As indoor users may pay more attention to relative location (in which buildings or room they are currently located versus absolute coordinates), an additional challenge might be how to display exact indoor locations (what type of information to provide users). In addition, converting numerical coordinates to a semantic representation, which usually includes a hierarchy of places (building, floor, room or country, state, city), might lead to a different kind of location information uncertainty (Hazas, et al., 2004). Also, it is essential to consider the availability of basemap information. As an extensive representation of a relatively larger space a basemap can only attain a certain level of accuracy (it is impossible to measure 
every feature inside the building) (Deitrick \& Edsall, 2009). In addition, the existing literature points out that map extent, map scale, and level of map generalization will influence a user's ability to utilize basemap information (Dillemuth, 2005; Dillemuth, Goldsberry, \& Clarke, 2007). These limitations of basemap detail and accuracy can also restrict the information that users can obtain from the system, which further impact users' opinions of location accuracy.

\subsubsection{Handling Location Uncertainty}

Deitrick and Edsall (2009) pointed out that uncertainty in geographic information causes "a mismatch between the needs and goals" of users and information producers. For positioning systems, this mismatch could result from users' limited knowledge or an unclear explanation of the location results, which in turn, would both increase decision making time and reduce their confidence in the system. Several studies have investigated users' struggle with location uncertainty in location-based games (Benford et al., 2003; Benford et al., 2006). However, the test bed of these experiments is limited to location-based gaming, which is quite different from real world use of location information. Research on real-world positioning has concentrated on using the users own experience to enhance the reliability of localization results, which further improves usability. For example, Sayda (2005) had hikers and mountaineers collecting trail data and updating positioning information about landmarks; this approach utilizes the number of users who gather data on the same object to evaluate the reliability of the data gathered by the users. Similarly, a correction-based mapping method (Bhasker, Brown, \& Griswold, 2004) based on user feedback has been used to improve the accuracy of indoor positioning. This improvement reduces the workload involved in building a database before calculation. 
In addition, researchers have considered positioning source data in terms of improving accuracy. For example, a visual inspection of each router is performed to ensure database entries are correct before the system is implemented (Bell, et al., 2010), which avoids the input of inaccurate source data from public users.

\subsubsection{User Opinion of Location-based Systems}

\subsubsection{System Usability Evaluation}

All positioning systems present users with some level of uncertainty (indoor, GPS, etc.). There might be conditions under which users cannot tell an inaccurate location from an accurate location; such a situation might be irrelevant as the two locations are very close to one another. However, in other situations the difference might be larger and meaningful, and the user shouldn't use the location information presented. As a result, there is a growing body of research investigating users when interacting with positioning systems to optimize the usability of the navigation tools. Since it is logical that users do not have the patience to deal with a poorly designed positioning system (Olsen, 2009), it is essential for positioning systems to have a user-friendly interface that facilitates users' needs. Designers must remember that most users of these systems are non-experts, which means the system should be highly interactive, one focus germane to the current research is the delivery of inaccurate location information. When a system is not working properly it should have the ability to communicate information about the calculated location (Gorlenko \& Merrick, 2003). Ciavarella \& Patern (2004) have discussed design issues of other currently available applications and then applied those beneficial design criteria to improve system usability in a museum environment from the perspective of users' needs for positioning information. Chincholle et al. (2002) have designed three routing tasks for users to perform in order to evaluate the 
usability of a specific navigation tool. Results from these tasks reflect users' attitudes towards the system, which to some degree reveals design deficiency. This kind of research provides empirical knowledge regarding users' preferences for building location-based systems.

\subsubsection{System Accuracy Evaluation}

When interpreting positioning results, users need to build a cognitive map, using their own spatial knowledge to locate themselves in reality and on the basemap. However, individual differences exist even under similar conditions, and users bring

their own knowledge and preferences to their judgments of system accuracy (Bell, et al., 2011). As a result, the speed and accuracy of the judgment process is highly dependent on a user's local knowledge, spatial ability, and confidence. For instance, researchers have concluded that users prefer to use a map oriented to their direction of travel when using a positioning system to find their way (Bornträger et al., 2003), a finding consistent with paper maps used in You-Are-Here scenarios (Thorndyke \& Hayes-Roth, 1982). Also, technical awareness (knowledge of the positioning technology) to some degree provides an opportunity to increase understanding when accuracy is low. If a user knows the principles of positioning calculation, their tolerance for positioning error could be higher than someone without this knowledge (Bell, et al., 2011). In addition, users having complete knowledge of the local environment tend to ignore the system's actual performance, which results in an inappropriate accuracy judgment (Stanton, Ragsdale, \& Bustamante, 2009). Similarly, users tend to be critical of system performance when they are highly confident and have abundant knowledge of their surroundings (Kantowitz, Hanowski, \& Kantowitz, 1997). When assessing system 
performance users' own characteristics, for instance, personal navigation experience, individual differences, and spatial abilities should be considered.

\subsubsection{User Trust of Location-based Systems}

\subsubsection{Definition and Impact of Trust}

Trust is a relatively new concept to the broad field of geographic information. However, it has been widely used for evaluating the usability of computer programs and related technology; these include trust of web-based services (Artz \& Gil, 2007; Flavián, Guinalíu, \& Gurrea, 2006; Roy, Dewit, \& Aubert, 2001) and collaborative computing systems (Thirunarayan, Anantharam, Henson, \& Sheth, 2010). Trust has been defined differently by researchers depending on the entity to be trusted, the definition of the entity, and user characteristics (Lee \& See, 2004). For positioning systems, Bell et al. (2011) defined it as "a user's opinion of the positioning results, which affects their adoption of and commitment to the system and their use of the information it provides." Trust can be increased by improving perceived accuracy (from a user's perspective), which not only requires an accurate calculation with high quality data, but also a clear interface that communicates position and uncertainty (or accuracy) (Bell, et al., 2011). Trust is an essential consideration for human computer interaction; research suggests there are many variables that affect user trust in computational settings (Fogg \& Tseng, 1999). Research on in-car navigation systems indicates that accuracy affects users' opinions of system credibility as well as their attitudes to the car (Jonsson, Harris, \& Nass, 2008; Jonsson, Harris, Nass, \& Takayama, 2005). Generally, users who trust the system have positive interactions, while users who lack trust behave in a negative manner (Wagealla, Terzis, \& English, 2003). Interestingly, it was found that user trust increases with risk, that is to say, the user still trusts the system even as it puts them at 
risk (Perkins, Miller, Hashemi, \& Burns, 2010). This finding is related to the system's role in a user's search for solutions to problems (such as being lost) and our increasing reliance on external solutions as problem complexity increases and our personal resources prove inadequate (Ishikawa, Fujiwara, Imai, \& Okabe, 2008).

\subsubsection{Dynamic Nature of Trust Change}

Common sense tells us users will trust accurate results more than inaccurate. However, this assumption is an over simplification of the matter, as system trust is not re-established each time a system calculates and communicates position, but is shaped over time based on experience with a system, system accuracy, and system reliability (Antifakos, Kern, Schiele, \& Schwaninger, 2005). In addition, a user may initially trust a computational system, only to distrust it later; it is also possible for a user to regain trust after initially distrusting a system (Tseng \& Fogg, 1999). Under certain circumstances, users may stop using a positioning system if they think the location information provided by the system is untrustworthy. Moreover, since the reliability of a system might not immediately affect user trust, it seems safe to suggest that a user's trust of a system is dynamic, ongoing, and complicated (Lee \& See, 2002). Specifically, trust is not only related to one-time accuracy, but also to the characteristics of the inaccuracy. This observation is supported by research on the reliance of automation, which suggests that both error type and error distribution have an impact on users' judgments of the automation (Sanchez, Rogers, Fisk, \& Rovira, 2011). The experiment results indicate that users still consider the automation alarms useful if the automation always provides alarms earlier than necessary (defined as "false alarms"); however, they abandon the automation if it fails to provide alarms during the whole process (defined as "miss"). Interestingly, users still check the information provided by the automation if it fails to 
provide alarms (“miss”) for a while, but they cease checking if the automation provides continuous "false alarms." If applied to positioning, "miss" cases are synonymous with inaccurate positioning, and occur if the system encounters signal problems (weak or insufficient signals). In fact, defining a "miss" is not straightforward, a single positioning result could be inaccurate to one user and accurate to another. In the latter case, it is likely the user would consider the result "good enough." As positioning is a continuous process occurring in real time, system performance is different across locations. In addition, users' confidence in a system could be affected by experience. As researchers suggest, participants are more likely to continue using route planning when the system has a lower initial error rate (Vries, Midden, \& Bouwhuis, 2003). Similarly, for an indoor positioning system, users may stop using that system in the future because of previous positioning inaccuracy.

\subsubsection{Problems and Gaps}

Undoubtedly, the spread of indoor positioning technology will prove a convenient service for mobile devices users. However, positioning information provided by those systems is far from reliable or ubiquitously accurate, as system performance is highly dependent on calculation method and source data. In addition, cost issues must be balanced when increasing system accuracy. Failure to achieve or maintain claimed accuracy could disappoint users who have high expectations of the system's capability. Such inconsistency in accuracy could have a substantial impact on a user's response and future use of such information. For example, users may feel frustrated if the system leads them in the wrong direction, but they are unlikely to pay attention to the software or hardware problems that cause inaccuracy. Given the challenges from both the user and the system, users' preferences and attitudes towards the system must be taken into 
consideration. This knowledge can be used to predict users' behaviour in their use of a specific positioning system or service, which, in turn, can contribute to a more usable positioning system design.

In order to provide users of indoor positioning systems with high usability, researchers have focused on improving positioning accuracy and evaluating absolute accuracy (distance between the two locations) between estimated location and actual location. These efforts are based only on one-time or average positioning accuracy and fail to consider the dynamic interaction between the user and device/system. Moreover, attempts have also been made to improve system usability and handle location uncertainty. However, user trust has not been explored, especially in instances when the system behaves inconsistently. Researchers have already pointed out that trust plays an essential role in human centered systems; furthermore, they have also noted that user trust is affected by experience with the system during the entire user experience with the system. For indoor positioning systems with high variability in accuracy, little research has investigated how this dynamic process affects a user's later trust of a positioning system. As a result, research still needs to be done on how localization results in different accuracy levels, as well as how the sequence of the results, affect users' trust of an indoor positioning system. This raises a series of questions regarding user trust of indoor location information:

1. How does user trust vary with positioning accuracy? As accuracy changes over time across multiple locations, it is important to know how users' opinions of inaccurate locations are different from accurate locations, especially when they get locations outside the correct building's boundary. 
2. How does previous experience with a positioning system affect user trust? The answer to this question will help explain how users' attitudes towards the system change.

3. Is system trust the same as positioning trust? Users' opinions of individual results are concentrated on performance at that specific moment in terms of position accuracy; however, their attitudes towards the system are formed in a more complicated way, which might be affected by previous experience of the system, the interface, and the data presented with the results through the interface.

4. What is the impact of user characteristics on trust? Parameters like personal navigation experience, familiarity with the environment, understanding of the positioning technology and spatial abilities should be considered when evaluating trust change. 


\section{METHODS}

\subsection{Participants}

54 students (27 males and 27 females) between the ages 19 to 32 (Mean: 23.56, SD: 3.04) participated in the experiment during the fall term of 2011. Using human participants in this experiment was approved by the Behavioral Research Ethics Board of the University of Saskatchewan in March, 2011. Students recruited from a geography course were given bonus credit; others were given an honorarium after the experiment. There were 18 participants in each of three experimental groups (described below), the number of males and females in each group were equal. All participants used in the study had some experience with the university campus.

\subsection{Materials}

\subsubsection{Software}

As WiFi signals are dynamic (even when a sensor is stationary (Xiang et al., 2004)), the positioning results calculated by our existing system (Saskatchewan Enhanced Positioning System (SaskEPS)) fluctuate. While accuracy is high (for one location the error will range from 4 to 7 meters, with the calculated location shifting slightly, as with GPS) using such results would mean each participant would be rating a slightly different positioning result. It is therefore impossible to completely control the positioning error of the presented location (result location). In this study, the positioning error was too important as an independent variable to be allowed to fluctuate (all participants should experience the same amount of error during each trial). Therefore, a simulated positioning system was used instead of our existing positioning system. From an interface perspective the simulated system functioned like 
the existing system. With a basemap including building shapes, hallway outlines, and roads on campus, the simulated system presented the calculated locations (predetermined points) on top of the basemap in the form of a green dot with green lines indicating the outline of the hallway in our experimental area (see figure 1 in section 2.5). Participants were not aware that locations were pre-determined or simulated. SaskEPS is embedded within SaskEXP (the experimental design software) running on an iPad. SaskEXP integrates the Santa Barbara sense of direction survey (Hegarty, Richardson, Montello, Lovelace, \& Subbiah, 2002), two spatial ability tasks, as well as SaskEPS; it also allows the researcher to control the display sequence of calculated locations based on designed experimental groups which will be illustrated later.

\subsubsection{Environment}

The experiment was conducted on the second floors of three connected buildings on campus (Kirk Hall, Agriculture Building, and Engineering Building). Both the complexity and connectivity of the buildings were considered when selecting the experiment buildings. Kirk Hall is the location of the Department of Geography and Planning, which has a relatively simple structure with only two hallways; most students involved in a geography course had some experience with it. The Engineering building, on the other hand, is one of the most complicated buildings on campus, with which students not enrolled in Engineering have limited experience. The second floor of the Agriculture building connects Kirk Hall and the Engineering building, which was beneficial for reducing travel time among buildings. In addition, the complexity of Kirk Hall, Agriculture building, and Engineering building ranges from simple to complicated, which allowed for the examination of user trust in various environments. 


\subsubsection{Survey}

The survey included two parts; the first part asked for general information about the participant and was presented in paper form. Besides their age and gender, this survey addressed participants' experience with the experiment environment and the positioning tool. For example, they were asked to rate their familiarity with Kirk Hall, the Agriculture Building, and the Engineering Building in a 7 option likert scale (1 indicates "not at all familiar," 7 indicates "very familiar") as well as their experience with positioning systems and positioning technology ( 1 indicates "nothing," 7 indicates "a lot"). The Santa Barbara Sense of Direction Scale (SBSOD) was used as the second part of the survey and was completed on an iPad, which included 15 statements regarding participants' "spatial and navigational abilities, preferences, and experiences" (Hegarty, et al., 2002).

\subsubsection{Spatial Ability Tasks}

Both of the spatial ability tasks were conducted on an iPad organized within the experiment design software SaskEXP. Verdine's Mental Rotations Task (MRT) was used as the first spatial ability task, which is used to examine participants' ability to mentally rotate an object in their head (Verdine, 2011). Participants were given an example question before the MRT tasks began. Once participants were familiar with the questions, they were asked to identify if the two shapes could be rotated to exactly match each other (by pressing "same" if they could and "different" if they could not). Each participant was required to complete 50 questions as accurately as possible, as there was no time limit for each question and they were allowed to come back to change the answer at any time before submitting all the answers. The total score for MRT was calculated and recorded in the log file, one point was give if they answered the question 
correctly. The second psychometric test was the Object Location Memory Test (OLMT) from Irwin and Marion (1992). Each participant was asked to view a screen including 27 objects and study the location of all the objects. After 60 seconds, they were shown a second screen including the same objects as the first screen. Participants were told that about half of the objects had been moved, and were asked to identify those objects by clicking on them; they were given another 60 seconds for this part of the task. One point was added to the final score if participants recognized one object that had been moved or was still in the same place correctly. Both MRT and OLMT scores were available to participants by request after completion of the experiment.

\subsection{Experiment Data}

85 experimental points (visited by participants in real time) were randomly generated in ArcGIS using building hallways (Kirk Hall, Agriculture Building, and Engineering Building) as a constraint. These locations represented trials in one of three "location accuracy" categories: 1. ACCURATE Locations (25 locations): these locations represent places for which the system provides accurate location information (the presented point within 5 meters of its actual position); 2. INACCURATE Locations (25 locations): these locations are inaccurate in absolute terms (positioning error is larger than 15 meters) but accurate in nominal/relative location (correct building); 3. WRONG BUILDING Locations (25 points): these locations are inaccurate in nominal terms (outside the correct building's boundaries); and 4. CONTINUOUS Locations (10 points): the positioning error of these locations ranges from 6 to 15 meters (one each at 1 meter intervals from 6 through 15 meters). 10 ACCURATE, 10 INACCURATE, and 10 WRONG BUILDING locations were used as priming locations for three groups respectively, the remaining locations in each category made up 55 post-priming locations which were 
used in all three groups followed by priming locations. Based on the hallway area of the three buildings, 17 points were selected from Kirk Hall, 34 from the Agriculture Building, and 34 from the Engineering Building. Similarly, the number of ACCURATE locations, INACCURATE locations, WRONG BUILDING locations, and CONTINUOUS locations in each building were based on the same ratio (as shown in table 1). Experimental locations (the positions presented to participants) were selected to be reasonable to participants (in the hallway or in a location outdoors where someone could stand, etc.).

Table 1. Four types of locations used in the priming and post-priming stage in three buildings ("K" represents Kirk Hall, "A" represents the Agriculture Building, and "E" represents the Engineering Building).

\begin{tabular}{cccccc}
\hline & & K & A & E & $\begin{array}{c}\text { Sum by } \\
\text { Category }\end{array}$ \\
& & & & & \\
\hline \multirow{2}{*}{ ACCURATE } & Priming & 2 & 4 & 4 & 10 \\
& Post-priming & 3 & 6 & 6 & 15 \\
INACCURATE & Priming & 2 & 4 & 4 & 10 \\
& Post-priming & 3 & 6 & 6 & 15 \\
WRONG BUILDING & Priming & 2 & 4 & 4 & 10 \\
& Post-priming & 3 & 6 & 6 & 15 \\
CONTINUOUS & Post-priming & 2 & 4 & 4 & 10 \\
\multicolumn{2}{c}{ Sum by Building } & 17 & 34 & 34 & Total: 85 \\
\hline
\end{tabular}

Each experimental point was moved in order for it to fall within its designated accuracy category (this was accomplished by applying a buffer equivalent to the inaccuracy necessary (less than $5 \mathrm{~m}$, more than $15 \mathrm{~m}$, etc.). For WRONG BUILDING locations, each experimental point was moved outside the building outline using the actual location as a reference. 


\subsection{Experimental Design}

Each participant visited a total of 65 locations in series: 10 priming locations from a single accuracy category followed by 55 post-priming locations (10 CONTINUOUS locations plus 45 locations across the three accuracy categories). Group 1 began with 10 ACCURATE priming locations; Groups 2 and 3 began with 10 INACCURATE priming locations and 10 WRONG BUILDING priming locations, respectively. A Latin Square design was used to establish visiting sequences of priming and post-priming locations within each building (to avoid moving back and forth among buildings), which is shown as table 2. In order to improve data collection efficiency, the internal visiting sequence of both priming and post-priming locations for each building was based on a clockwise or counter-clockwise direction.

Table 2. Building visiting sequences for each group.

\begin{tabular}{llllllll}
\hline & \multicolumn{4}{c}{ Priming Locations } & \multicolumn{2}{c}{ Post-priming Locations } \\
\hline \multirow{3}{*}{ Group 1 } & a & K & A & E & E & A & K \\
& b & A & E & K & K & A & E \\
& c & E & K & A & A & E & K \\
& a & K & A & E & E & A & K \\
& b & A & E & K & K & A & E \\
& c & E & K & A & A & E & K \\
& a & K & A & E & E & A & K \\
& b & A & E & K & K & A & E \\
& c & E & K & A & A & E & K \\
\hline
\end{tabular}

Finally, minor modifications were made for the following reasons: 1. Locations near one another were presented with similar accuracy, as a user might expect from a 
real system; 2. The number of locations presented between two WRONG BUILDING locations was not constant, as such a pattern might be evident to participants. In this way, each group allowed for the examination of how accuracy at previously visited locations (accuracy of priming locations) affects subsequent trust evaluation (trust of post-priming locations). Furthermore, the overall body of data allowed for an examination of how different levels of accuracy (ACCURATE, INACCURATE, WRONG BUILDING) affect trust.

\subsection{Procedures}

Before the experiment, each participant was randomly assigned to one of the nine sub groups mentioned above (including building visiting sequence) with an anonymous participant ID. Accordingly, SaskEPS was set to display positioning results in the appropriate sequence. The experiment took approximately 100 minutes, and all participants did the experiment individually with the researcher's guidance. Scheduling was completed using the Doodle online meeting-scheduling tool. Each participant finished the experiment in the following three steps: survey, spatial ability tasks, and positioning tasks.

Each participant was initially met in a meeting room in Kirk Hall. After reading the consent form, they were free to sign and participate in the experiment. Once signing the consent form, participants were given brief instructions about the experimental procedures. Participants were allowed to ask questions at any time to avoid confusion in the experiment process.

They were then asked to complete the survey (familiarity with buildings, GPS experience and Santa Barbara Sense of Direction Scale). After finishing the survey, participants were asked to complete two spatial ability tasks (mental rotation task and 
Object Location Memory Test); instructions were provided before they began the survey and spatial ability tasks. After a short training session focusing on how the positioning interface and program works, participants were asked to perform positioning tasks at 65 experimental sites, the visiting sequence of which was decided according to one of nine sub conditions above. At each point, participants were asked to rate their confidence in the positioning results (defined as "position trust") and the positioning system (defined as "system trust") (Figure 1).

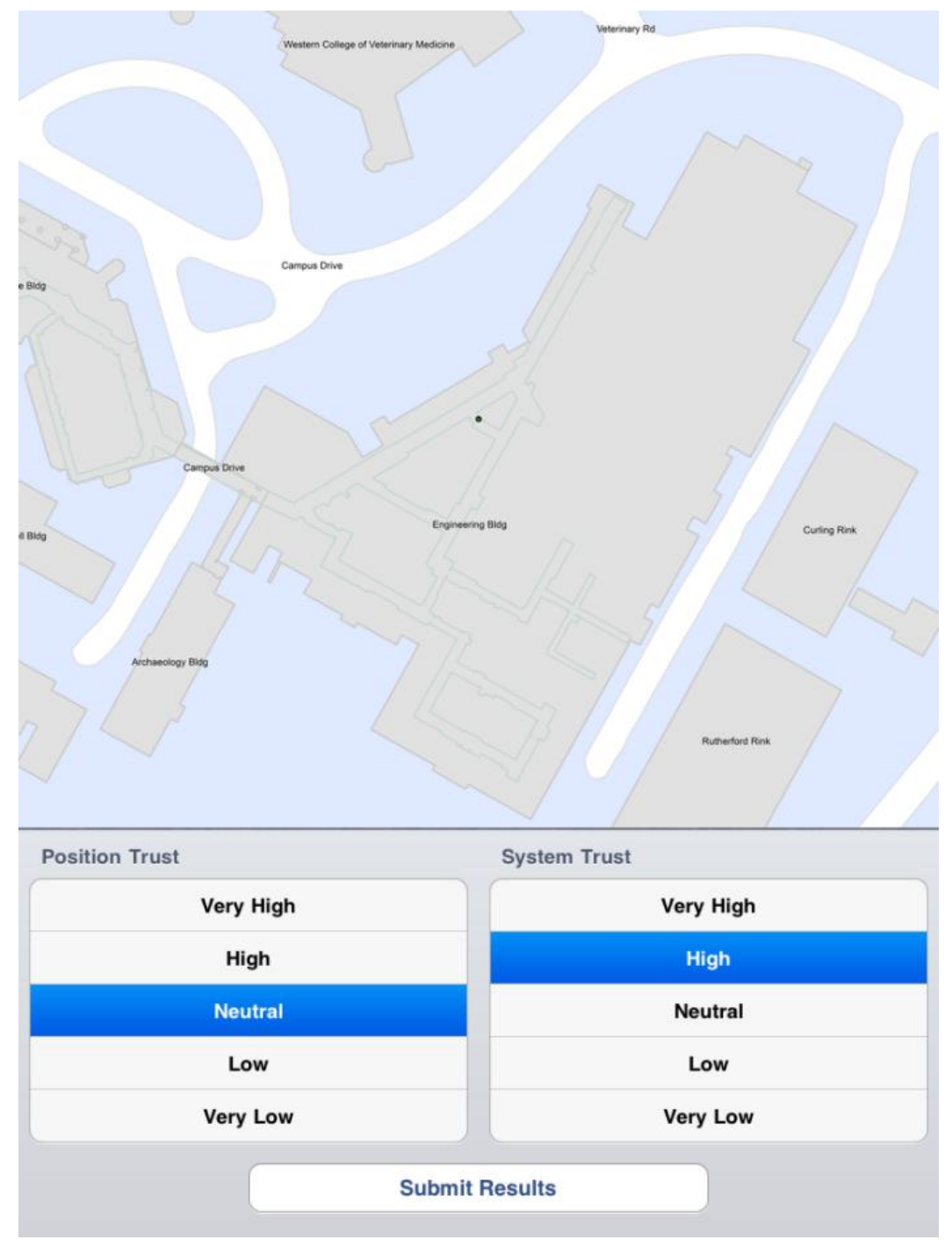

Figure 1. Data recording interface of the simulated SaskEPS 
"Positioning trust" illustrates how much they trust the position calculated at an individual location by the positioning system, while "System trust" represents their confidence in the system overall. Both trusts were evaluated on a 5-point likert scale (5="Very high," 4="High," 3="Neutral," 2="Low," and 1="Very low"). Participants used their own judgments when providing "Position trust" and "System Trust" ratings as they were told that there was no standard measurement for their confidence in the system or positioning results. In addition, participants were encouraged to verbally provide their reasons for each trust rating (these comments were recorded by the researcher). As the system was not calculating location in real time participants were informed to initiate positioning only when they were at the designated experimental site (they were told the data was only needed at designated experimental sites for this research). 


\section{III}

\section{RESULTS}

Dependent variables obtained from the experiment include: Two types of trust at each location (positioning trust and system trust). The ancillary data collected before the positioning tasks are considered as independent variables, which include the total score for Mental Rotation Task, total score for Object Location Memory test, average score for the Santa Barbara Sense of Direction Scale (SBSOD), familiarity with experimental environment, as well as GPS knowledge and experience.

\subsection{Position Trust}

Analysis regarding position trust was broken down into two tests: 1. Between positioning accuracy types (ACCURATE, INACCURATE, and WRONG BUILDING) and 2. Between priming groups; the latter was examined by looking within accuracy types between groups. The ordinal data from position trust ratings were not normally distributed, violating an essential assumption of the preferred test, Multivariate Analysis of Variance (MANOVA) (transformations failed to normalize the data). As a result the Kruskal Wallis $H$ test was used to examine position trust variation in different accuracy categories (ACCURATE, INACCURATE, and WRONG BUILDING); this analysis examined only the 55 post-priming locations and the impact of priming location accuracy (starting with ACCURATE (G1), INACCURATE (G2), WRONG BUILDING priming locations (G3)) on users' trust of ACCURATE, INACCURATE, and WRONG BUILDING locations in these trials. If the main effect of accuracy category was significant, the Mann-Whitney $U$ Test was used for paired comparisons. Bonferroni corrections were applied to adjust the pre-chosen significance level $(\alpha=0.05 / 3=0.0167)$ to make the significance test more stringent. 


\subsubsection{Overall Results}

Table 3 provides descriptive statistics for position trust ratings of post-priming locations, PT_A represents position trust for ACCURATE locations, PT_IA represents position trust for INACCURATE locations, and PT_WB represents position trust for WRONG BUILDING locations. In general and as expected, ACCURATE locations generate much higher trust than INACCURATE locations; WRONG BUILDING locations were the least trusted (Kruskal-Wallis $H$ test results $(H=36.054$, $\mathrm{df}=2, p<$ o.001)); paired comparisons using the Mann-Whitney $U$ Test were also significant ( $p<$ o.oo1 for each pair). In addition, users' opinions of INACCURATE locations show more variance than that of ACCURATE locations and WRONG BUILDING locations (evident from standard deviation and between group variance).

Table 3. Descriptive statistics for Position Trust (18 participants in each group, which includes 15 ACCURATE, 15 INACCURATE, and 15 WRONG BUILDING locations after priming locations).

\begin{tabular}{|c|c|c|c|c|c|c|c|c|c|c|}
\hline \multicolumn{2}{|c|}{ Trust } & \multicolumn{3}{|c|}{ PT_A } & \multicolumn{3}{|c|}{ PT_IA } & \multicolumn{3}{|c|}{ PT_WB } \\
\hline & & $\begin{array}{l}\text { Mean } \\
\text { (SD) }\end{array}$ & Min & $\operatorname{Max}$ & $\begin{array}{l}\text { Mean } \\
\text { (SD) }\end{array}$ & Min & $\operatorname{Max}$ & $\begin{array}{l}\text { Mean } \\
\text { (SD) }\end{array}$ & Min & Max \\
\hline \multicolumn{2}{|c|}{ Overall } & $\begin{array}{c}4.46 \\
(0.30)\end{array}$ & 3.55 & 4.83 & $\begin{array}{c}2.21 \\
(0.58)\end{array}$ & 1.11 & 3.72 & $\begin{array}{c}1.57 \\
(0.41)\end{array}$ & 1.05 & 2.67 \\
\hline \multirow{3}{*}{$\begin{array}{l}0 \\
0 \\
0 \\
0\end{array}$} & G1 & $\begin{array}{c}4.22 \\
(0.09)\end{array}$ & 3.55 & 4.72 & $\begin{array}{c}1.83 \\
(0.11)\end{array}$ & 1.11 & 2.78 & $\begin{array}{c}1.30 \\
(0.05)\end{array}$ & 1.05 & 1.55 \\
\hline & G2 & $\begin{array}{c}4.53 \\
(0.06)\end{array}$ & 3.94 & 4.83 & $\begin{array}{c}2.04 \\
(0.10)\end{array}$ & 1.55 & 2.67 & $\begin{array}{c}1.46 \\
(0.07)\end{array}$ & 1.05 & 2.17 \\
\hline & G3 & $\begin{array}{c}4.63 \\
(0.04)\end{array}$ & 4.33 & 4.78 & $\begin{array}{c}2.77 \\
(0.13)\end{array}$ & 2.05 & 3.72 & $\begin{array}{c}1.94 \\
(0.11)\end{array}$ & 1.33 & 2.67 \\
\hline
\end{tabular}




\subsubsection{Priming Effect on Position Trust}

The next step was to examine the role that priming plays in position trust. Recall that ACCURATE priming locations were expected to increase subsequent position trust. In fact, the opposite is true. The Kruskal-Wallis results indicate that for Group 1, participants who were primed with 10 ACCURATE positioning results, position trust ratings for all three types of locations (PT_A, PT_IA, and PT_WB) are significantly different (lower) than ratings by participants who were primed with 10 INACCURATE or 10 WRONG BUILDING locations (comparisons among groups for post-priming ACCURATE locations: $H=15.025, \mathrm{df}=2, p=0.001$; for post-priming INACCURATE locations: $H=19.617, \mathrm{df}=2, p<0.001$; and for post-priming WRONG BUILDING locations: $H=18.328, \mathrm{df}=2, p<0.001$ ). Mean values of trust for the three types of locations across groups are shown in figure 2. The overall pattern indicates that users' trust of the three location types is similar across groups. However, priming with WRONG BUILDING locations (Group 3) has a stronger effect on increasing later position trust than INACCURATE priming (Group 2). 


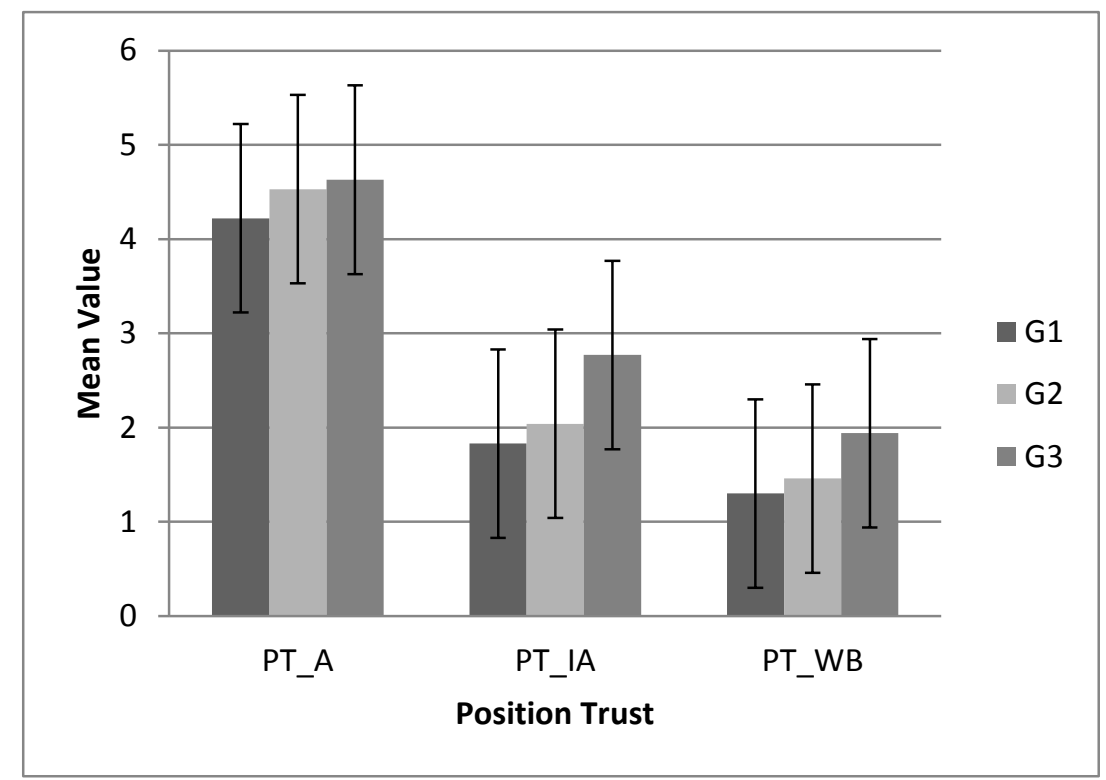

Figure 2. Mean values of position trust of three location types (error bars: +/-1 SE) (PT_A: position trust of ACCURATE locations, PT_IA: position trust of INACCURATE locations; PT_WB: position trust of WRONG BUILDING locations; Groups, G1: ACCURATE priming, G2: INACCURATE priming, G3: WRONG BUILDING priming),

The pair-wised comparisons between groups for position trust of each type of location were examined using the Mann-Whitney $U$ Test. Calculated $p$ values can be found in Table 4 (* indicates significant at 0.0167 level). For ACCURATE locations users have greater trust if they start with WRONG BUILDING locations and INACCURATE locations than when starting with ACCURATE locations. Regarding INACCURATE and WRONG BUILDING locations, starting with WRONG BUILDING locations results in higher trust compared to groups that start with ACCURATE and INACCURATE locations. In addition, priming with WRONG BUILDING locations produces the highest ratings of trust. 
Table 4. Calculated $p$ values from the Mann-Whitney U Test.

\begin{tabular}{cccccccccc}
\hline & \multicolumn{3}{c}{ PT_A } & \multicolumn{3}{c}{ PT_IA } & \multicolumn{3}{c}{ PT_WB } \\
& G1 & G2 & G3 & G1 & G2 & G3 & G1 & G2 & G3 \\
\hline G1 & - & $0.005^{*}$ & $<0.001^{*}$ & - & 0.161 & $<0.001^{*}$ & - & 0.161 & $<0.001^{*}$ \\
G2 & - & - & 0.187 & - & - & $<0.001^{*}$ & - & - & $0.002^{*}$ \\
G3 & - & - & - & - & - & - & - & - & - \\
\hline
\end{tabular}

Upon closer inspection, interesting patterns among types of locations and groups were discovered; these results are presented as a summary of significant differences in mean position trust ratings in table 5 . For ACCURATE and INACCURATE locations, the increase in trust occurs in groups when priming locations are in less accurate categories. For WRONG BUILDING locations, as they are in the least accurate category, the significant trust increase occurs in groups when priming locations are in the equivalent category (Group 3: WRONG BUILDING priming). It can be concluded that experiencing less accuracy first, especially WRONG BUILDING locations (strongest effect), has a positive impact on users' trust of positioning results that follow; conversely, when starting with ACCURATE priming locations trust in all following locations declines.

Table 5. Significant paired comparisons for position trust of three types of locations across groups.

\begin{tabular}{cc}
\hline & Mean value comparison (significant scenarios) \\
\hline PT_A & $\begin{array}{c}\text { Starting with WB (G3) and IA (G2) > Starting } \\
\text { with A (G1) }\end{array}$ \\
PT_IA & Starting with WB (G3) > Starting with IA (G2) \\
& and A (G1) \\
PT_WB & $\begin{array}{c}\text { Starting with WB (G3) }>\text { Starting with IA (G2) } \\
\text { and A (G1) }\end{array}$ \\
\hline
\end{tabular}




\subsubsection{Position Trust of Locations in the Same Category}

When comparing the position trust of locations in the same category between priming stage and post-priming stage (PT_A in both stages for Group 1, PT_IA in both stages for Group 2 and PT_WB in both stages for Group 3), independent samples $t$-tests (PT_WB are normally distributed after logarithmic transformation) were used for examining the significant mean differences of position trust across the two stages for ACCUATE, INACCURATE, and WRONG BUILDING locations. The mean values are included in Table 6, slight increases can be found in PT_A and PT_WB across two stages, on the contrary, PT_IA decreases a bit in the post-priming stage. However, none of those changes is statistically significant, which indicates that the priming locations do not have a significant impact on the post-priming locations falling within the same category as the priming locations.

Table 6. Descriptive statistics for position trust of ACCURATE, INACCURAE and WRONG BUILDING locations in both priming stage and post-priming stage.

\begin{tabular}{ccccccc}
\hline & \multicolumn{3}{c}{ Priming stage } & \multicolumn{3}{c}{ Post-priming stage } \\
& Mean (SD) & Min & Max & Mean (SD) & Min & Max \\
\hline PT_A in G1 & $4.10(0.12)$ & 3.10 & 4.90 & $4.22(0.10)$ & 3.53 & 5.00 \\
PT_IA in G2 & $2.41(0.15)$ & 1.11 & 3.50 & $2.04(0.11)$ & 1.20 & 2.93 \\
PT_WB in G3 & $1.82(0.16)$ & 1.00 & 3.1 & $1.94(0.14)$ & 1.00 & 2.87 \\
\hline
\end{tabular}

\subsection{Priming Effect on System Trust}

An Analysis of Variances (ANOVA) test was used to examine significant system trust difference in post-priming results across groups. Table 7 shows the mean values of system trust for each group, only system trust in Group 3 (WRONG BUILDING priming) is significantly higher than that in Group 2 (INACCURATE priming) $(\mathrm{F}(2,51)=4.018$, 
$p=0.024)$. The positioning system used in Groups 2 and 3 has the same reliability, in which participants experienced the same number of ACCURATE locations during the positioning process (including both priming stage and post-priming stage). It can be concluded that when system reliability is the same, seeing continuous inaccurate locations (WRONG BUILDING priming) will increase system trust. However, this effect is not significant for Group 1 (ACCURATE priming), as the overall reliability of system used in Group 1 is higher than that used in Groups 2 and 3.

Table 7. Descriptive statistics for system trust in post-priming stage.

\begin{tabular}{ccccc}
\hline & \multicolumn{3}{c}{ ST (Post-priming stage) } & \\
& & Mean (SD) & Min & Max \\
\hline \multirow{2}{*}{} & $\mathrm{G} 1$ & $2.74(0.38)$ & 1.93 & 3.31 \\
& $\mathrm{G} 2$ & $2.56(0.52)$ & 1.71 & 3.69 \\
& $\mathrm{G} 3$ & $2.95(0.30)$ & 2.47 & 3.40 \\
\hline
\end{tabular}

\subsection{Comparison between Position Trust and System Trust}

A within-subject test was used to compare the difference between average position trust and system trust ratings of all three groups. Trust type is considered as the within-subject factor as position trust and system trust are repeated measures assessed by the same participant; while location type is treated as the between subject factor (category factor). Table 8 provides descriptive statistics for average position trust and system trust for all three groups at ACCURATE, INACCURATE, and WRONG BUILDING locations. 
Table 8. Descriptive statistics for position trust (PT) and system trust (ST) at ACCUATE (T_A), INACCURATE (T_IA), and WRONG BUILDING (T_WB) locations in the post-priming stage.

\begin{tabular}{rrrrrrr}
\hline & \multicolumn{2}{c}{ PT } & & \multicolumn{3}{c}{ ST } \\
& Mean (SD) & Min & Max & Mean (SD) & Min & Max \\
\hline T_A & $4.46(0.30)$ & 3.94 & 4.78 & $3.21(0.15)$ & 2.92 & 3.52 \\
T_IA & $2.21(0.58)$ & 1.61 & 2.83 & $2.51(0.25)$ & 2.07 & 2.85 \\
T_WB & $1.57(0.41)$ & 1.17 & 2.13 & $2.19(0.15)$ & 2.00 & 2.55 \\
\hline
\end{tabular}

In general, ACCURATE locations generate higher position and system trust than INACCURATE locations, and WRONG BUILDING locations are the least trusted ( $p$ value $<0.001$ for each pair); the interaction is also significant. Specifically, position trust is higher than system trust at ACCURATE locations; while system trust is higher than position trust at INACCURATE and WRONG BUILDING locations (as shown in figure 3). These results indicate that participants are more confident about the position calculated at an individual location than the system itself when the system functions accurately. However, when experiencing inaccuracy, participants trust the system itself more than the inaccurate results. 


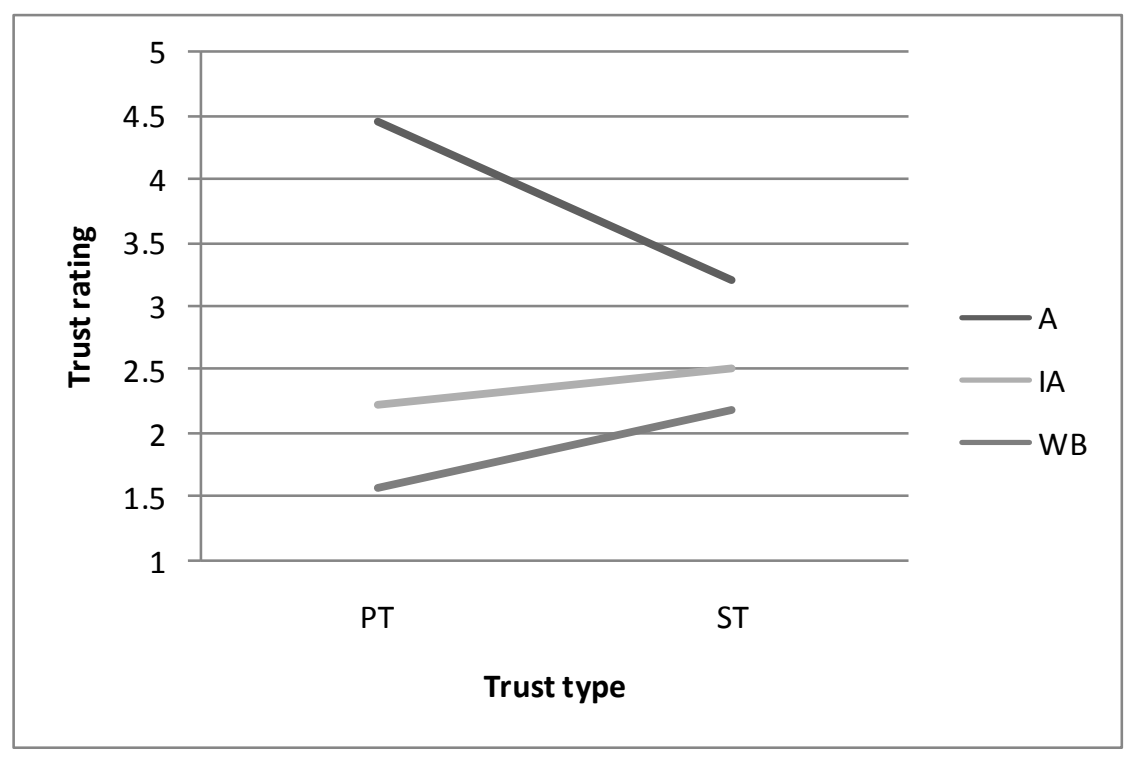

Figure 3. Mean values of position trust (PT) and system trust (ST) of three location types (A: trust of ACCURATE locations, IA: trust of INACCURATE locations; WB: trust of WRONG BUILDING locations)

In the post-priming stage, inaccuracy appears randomly during the experimental process. Thus, participants had alternating exposure to both accurate and inaccurate trials. In other words, they might experience, for example, several accurate locations first, then several inaccurate locations, followed by some accurate locations again. At both accurate and inaccurate locations, participants have reservations about the system's overall performance than at an individual location. As shown in figure 3 , system trust is much less variable than position trust. Specifically, a sharper decrease can be found in position trust than in system trust when inaccuracy occurs, as users need to see more results to confirm the system's ability, or inability, to produce accurate results. This difference results in a higher system trust rating than position trust rating at inaccurate locations. That is to say, when the system fails to maintain previously experienced accuracy, system trust drops more slowly than position trust. Therefore, it 
can be concluded that position trust is more directly affected by inaccuracy; system trust is more complex and is slower to change than position trust.

\subsection{Effect of Positioning Error on Position Trust}

According to figure 4, from an overall point of view, the trend shows a decrease in average position trust when positioning error increases. However, this association between the two variables is not perfectly linear. The average position trust fluctuates between the low of 1.67 and a peak of 4.83 , which may be attributed to the individual differences in location accuracy judgment. Here, WRONG BUILDING locations are not included in the figure below, as the characteristic that defines their inaccuracy is NOT distance from actual location but their positioning outside the building in which the participant is currently standing.

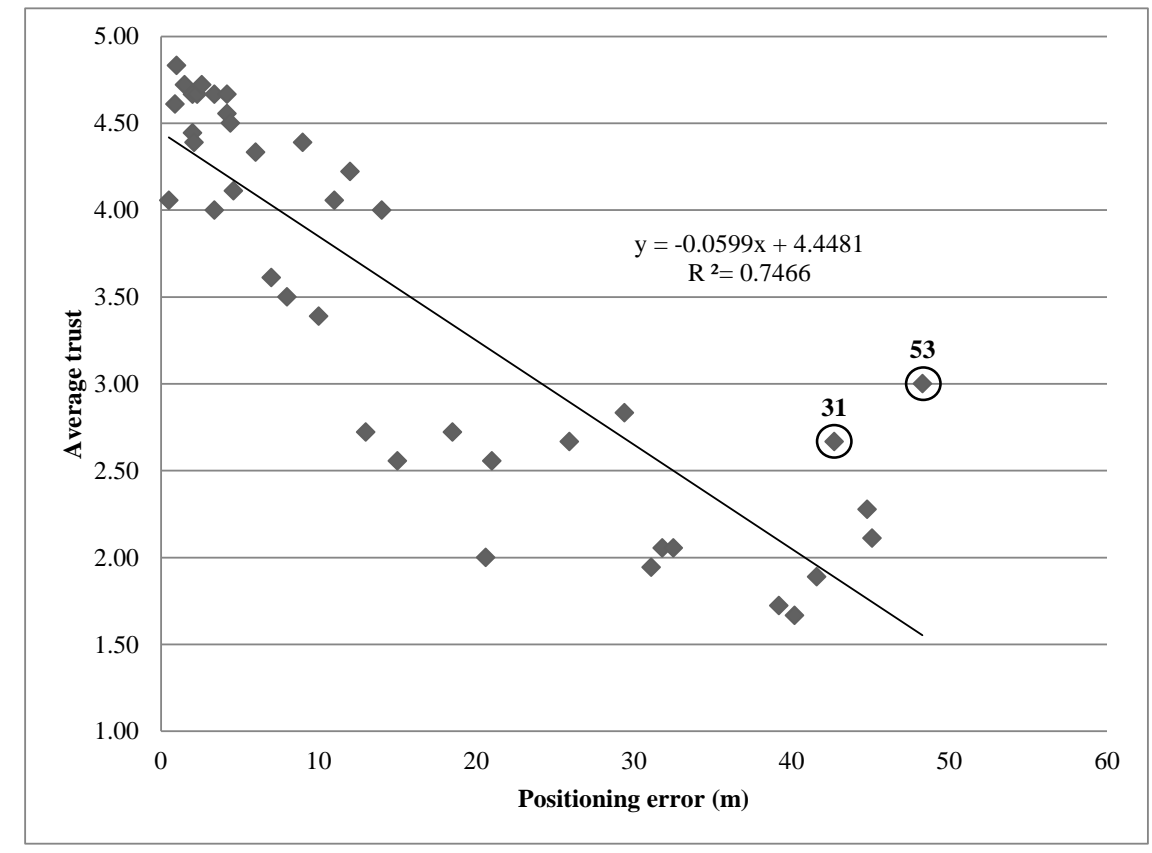

Figure 4. Relationship between positioning error and average position trust ratings of ACCURATE, CONTINUOUS and INACCURATE locations (40 points included, positioning error $<=50 \mathrm{Om}$ ) 
Furthermore, this negative association becomes stronger when increasing the number of inaccurate locations (evident from the increasing $\mathrm{R}$ square). As shown in table 9, there is a substantial increase in $\mathrm{R}$ square (from 0.03 to 0.46 ) when the positioning error increases from 5 meters to 10 meters, and this increase continues (from 0.46 to 0.83 ) until the positioning error is over 40 meters $(0.75)$.

Table 9. R square values of different linear models describing relationship between positioning error and average position trust ratings of ACCURATE, CONTINUOUS and INACCURATE locations.

\begin{tabular}{ccc}
\hline Total points included & Positioning error & R square \\
\hline 15 & $<=5$ meters & 0.03 \\
20 & $<=10$ meters & 0.46 \\
25 & $<=15$ meters & 0.53 \\
30 & $<=30$ meters & 0.72 \\
34 & $<=40$ meters & 0.83 \\
40 & $<=50$ meters & $0.75\left(0.82^{*}\right)$ \\
\hline
\end{tabular}

Note: * after removing two outliers.

Upon closer inspection, it can be found that participants' average position trust rating at two points (a shown in figure 4), which are over 40 meters from the actual location, are much higher than those points which have similar positioning error. After removing these two outliers, the $\mathrm{R}$ square value increases to 0.82 , which is more consistent with the overall pattern. Interestingly, the high position trust ratings at those two points result from a small number of participants choosing to trust the positioning results regardless of actual inaccuracy because the environment is complicated or confusing. For point 31, participants who gave a relatively high position trust rating explained that the surroundings of the points on the map seem to be similar with the 
real environment where they were standing. This is understandable, because the Engineering building is complicated and most participants have low familiarity with it. For point 53, where participants stand is almost the same location in the hallway as the presented position (in terms of hallway structure), as a result, it is not surprising that some participants treat them as the same point.

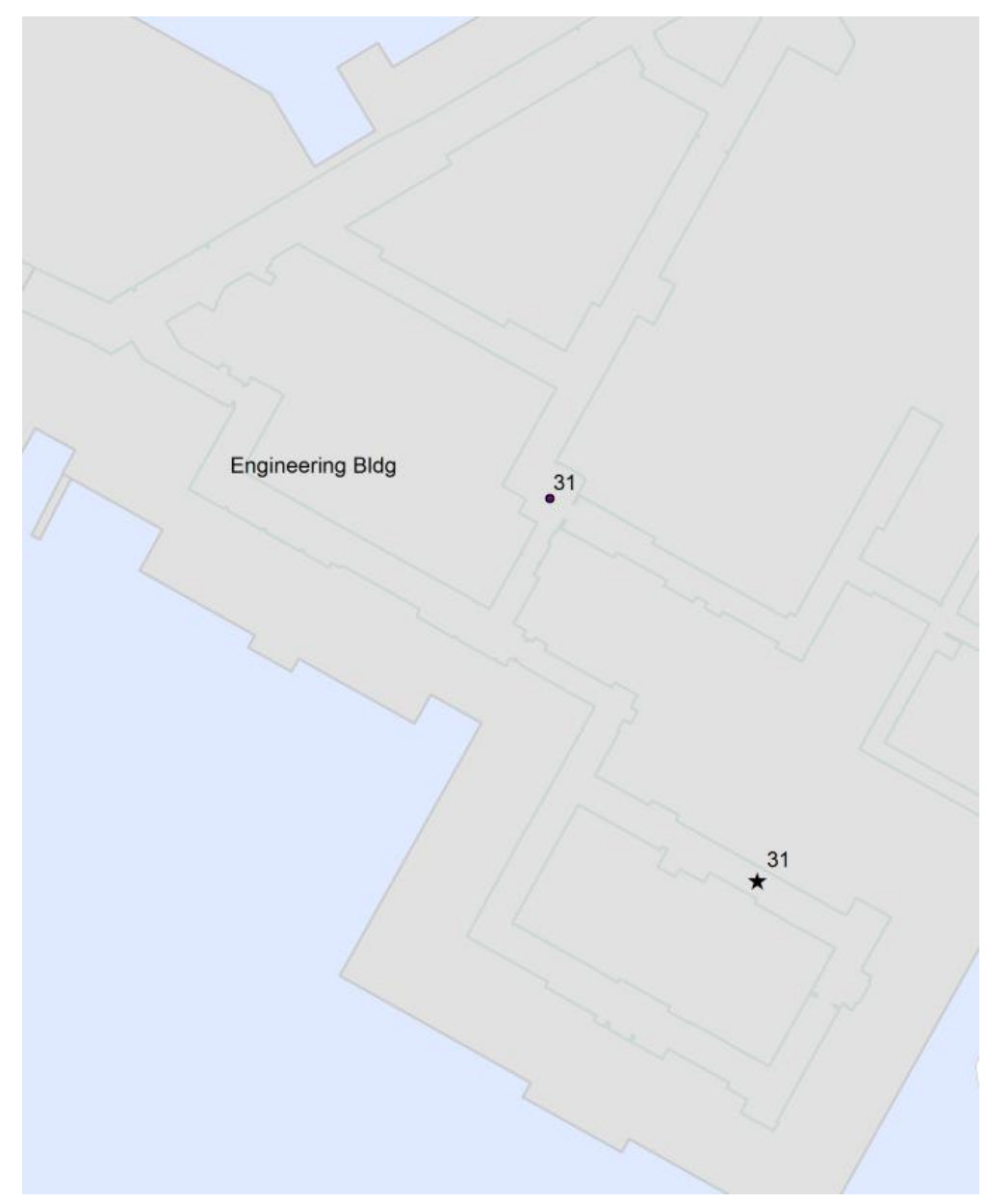


Figure 5. Actual (where participants stand) and result location (calculated by the system) of points 31 (top) and 53 (bottom) (black star represents the actual location and black dot represents the result location).

\subsection{Individual Differences}

Data collected from the survey and spatial ability tasks before positioning tasks is summarized in Table 10. According to the descriptive statistics, male participants have a slightly higher mean score on the Mental Rotation Task, while female participants perform better on the Object Location Memory Test and have a slightly better sense of direction. In addition, results suggest that male participants have more experience with the environmental area and GPS technology than women participants. However, results from independent samples $t$-tests indicate that gender difference is statistically significant only in Mental Rotation Task $(p=0.008)$. 
Table 1o. Descriptive statistics of data collected from the survey and spatial ability tasks by gender (MRT: Mental Rotation Task score; OLMT: Object Location Memory Test score; AVG_SBSOD: average score of SBSOD; SUM_Familiarity: sum ratings of familiarity with all three buildings; GPS_Experience: rating of GPS experience; GPS_Principle: rating of understanding of the GPS principle)

\begin{tabular}{cccccccc}
\hline & & MRT & OLMT & AVG_SBSOD & SUM_Familiarity & GPS_Experience & GPS_Principle \\
\hline Overall & AVG & 0.87 & 20.11 & 4.00 & 9.39 & 2.74 & 3.31 \\
& SD. & 0.11 & 2.70 & 0.35 & 3.24 & 1.83 & 1.26 \\
Female & AVG & 0.83 & 20.70 & 4.03 & 8.70 & 2.67 & 3.19 \\
& SD. & 0.11 & 2.81 & 0.34 & 3.02 & 1.44 & 1.11 \\
Male & AVG & 0.92 & 19.52 & 3.98 & 10.07 & 2.81 & 3.44 \\
& SD. & 0.11 & 2.49 & 0.36 & 3.35 & 2.18 & 1.40 \\
\hline
\end{tabular}

A correlation analysis was conducted to examine the relationships among the above variables and trust. From an overall point of view, participants who have a higher Object Location Memory score trust the system less, which is indicated by the negative significant correlation between OMLT score and average system trust ( $\mathrm{r}(54)=-0.382$, $p=0.004)$. This finding illustrates that people who are better able to passively remember places and locations are more confident about where they are and are less willing to trust positioning results that are not consistent with their own knowledge. When investigating the correlation for female and male participants separately, a different impact on each group is seen. For female participants, the positive significant correlation between GPS principle and trust in WRONG BUILDING locations ( $\mathrm{r}(27)=$ $0.420, p=0.029$ ) indicates that the knowledge of GPS principle helps them to put positioning error in context when the system displays the locations outside the correct building's boundaries. While for male participants, OMLT and MRT have a significant 
negative correlation with male participants' trust in the system $(\mathrm{r}(27)=-0.383, p=$ 0.049; $\mathrm{r}(27)=-0.386, p=0.047)$. Besides, OMLT and familiarity with the experimental buildings also have a significant negative correlation with male participants' trust in WRONG BUILDING locations ( $\mathrm{r}(27)=-0.427, p=0.026 ; \mathrm{r}(27)=-0.471, p=0.013$ ). These results are consistent with the overall findings; in addition, high familiarity with the environment reduces trust in WRONG BUILDING locations. A lack of significant correlation between average score of SBSOD and trust suggests that sense of direction does not particularly affect users' trust evaluation. In summary, high spatial ability and familiarity with the environment have a negative impact on users' trust, while understanding the GPS principle could positively affect users' trust. 


\section{IV}

\section{DISCUSSION}

\subsection{Positioning Accuracy VS. Trust}

For all three groups, position trust is substantially higher for ACCURATE locations. Users trust ACCURATE locations most, followed by INACCURATE locations, and WRONG BUILDING locations are the least trusted. Interestingly, beyond this result the position trust fluctuates widely even in the same accuracy category. This fluctuation is more obvious in CONTINUOUS locations, the positioning trust ratings of which do not decrease linearly with the increased positioning error (in one meter increments). That is to say, it is not necessarily true that a user would trust a position with 10 meters of positioning error more than the one with 20 meters of positioning error. Individual differences in accuracy judgment should be taken into consideration as well, as these factors might result in overconfidence in the system or generally by users themselves. However, it is important to note that the trust ratings of ACCURATE locations (within 5 meters of positioning error) cluster around 4.5 (as shown in figure 4) with less variation. When the positioning error exceeds 5 meters (CONTIOUOUS and INACCURATE locations), users' trust ratings are more varied and do not achieve the same level of trust as ACCURATE locations. This dividing line at 5 meters is also emphasized by the substantial increase in $\mathrm{R}$ square (0.03 to 0.46 as shown in table 9). Although in some cases users may give high trust ratings at WRONG BUILDING locations because of the

relatively lower absolute error (displayed location was outside, but could be relatively close to the user's actual location). However, the overwhelming pattern is that users trust locations within building outlines more than those outside building outlines.

These results are consistent with the preliminary hypothesis: for an individual 
location, better accuracy will generate higher trust. In other words, inaccuracy has a negative impact on trust. Therefore, designers should take measures to keep users engaged and not lose them to distrust during the period of inaccuracy. Besides, this negative effect depends on the characteristics of inaccuracy: if the location is outside current building's outline or not. In the case of WRONG BUILDING locations, it is essential to incorporate a constraint that would not let a position be displayed beyond a building's footprint. Since most devices that would be running such a system also include a GPS sensor, engaging this information is the best way to determine if the device is outdoors (if the system sees several GPS signals, some of relatively high strength, there is a high likelihood that the device is outdoors, otherwise it is NOT).

\subsection{Priming Effect on Trust}

Interestingly, the hypothesis regarding the impact of priming with locations in different types of accuracy was refuted by the results. Priming with inaccurate locations, especially WRONG BUILDING locations, can increase trust in later positioning results. Users appear to have higher expectations when they initially see a series of accurate locations. On the contrary, seeing inaccurate locations likely decreases users' initial expectations, making subsequent locations (particularly accurate locations) seem more trustworthy. One way of thinking of this pattern is that seeing consistently accurate results sets the bar high, subsequent inaccurate locations don't live up to expectations and are therefore rated as far less trustworthy. Nevertheless, this effect does not extend to locations that are as accurate as the priming locations within the same priming group. Compared to position trust, the priming effect on system trust is weaker, as it is only significant when system reliability (the percentage of ACCURATE locations in the entire 
positioning process) is the same. For Groups 2 and 3 (INACCURATE priming and WRONG BUILDING priming), the priming effect does not have a significant impact on increasing users' trust in the system during post-priming stage as compared to Group 1 (ACCURATE priming). This phenomenon suggests that the development of system trust is not only based on the one-time accuracy or improved accuracy at each individual location as compared to the previous locations but also the overall performance of the system. In other words, users take into account the system's ability to provide accurate locations through their full interaction with the system.

In real world settings, positioning and associated accuracy change continuously. Interpreting the above patterns suggests that a positioning system that initially accurate and then loses accuracy might cause users to distrust and abandon the system. Specifically, if a positioning system functions well for a period of time users will expect to have at least equivalent accuracy later. When system accuracy is low for a specific and possibly knowable reason, user trust will decline; this negative impact will likely extend to subsequently accurate results as well. To avoid such an outcome, indoor positioning systems should target consistent accuracy. Although the results suggest that trust can recover from inaccurate results, this resilience of trust should not be a reason to expect users will continue to use an inaccurate system. It is possible that most users will continue with a system with periodic and/or one-time inaccuracy, but each subsequent inaccurate location increases the likelihood that a user will stop using the system (and not return). One suggestion is to have the system provide information explaining inaccuracy (e.g. out of system range, not enough available signals, etc.) in order to reduce the inflation of expectations. This communication through GUI offers a good 
opportunity for users to regain trust before abandoning the system.

\subsection{Position Trust VS. System Trust}

The other question raised in this research is whether users treat system trust the same as position trust. Unlike individual positioning results, the system is made up of three components visible to the user: 1 . Overall interface, 2. Calculation process, and 3. Output positioning results. The first two are likely to mediate a decline in trust due to the relatively trustworthy nature of both the system's appearance during calculation and the provision of accurate basemap data when results are presented (if present). Results from the initial comparison between position trust and system trust at three types of locations gives a clear answer to this question. System trust has less variability and is less sensitive to inaccuracy compared to position trust. This pattern is further reflected by the development of trust across individual positioning results: the development of position trust occurs faster than system trust, which is attributed to users' overall trust in the system. When inaccuracy occurs, system trust changes less quickly than position trust. According to users' comments, they tend to believe that the occurrence of inaccuracy is periodic and the system will recover in subsequent results. Similarly, when the system behaves accurately after recovering from inaccuracy, they still wait for more accurate results to confirm the system's ability to provide accurate results. In other words, system trust is accumulated over time across multiple locations.

This lag in system trust change holds important clues for positioning system designers and researchers who are interested in human interaction with positioning systems. According to the results, users' system trust ratings become gradually lower as a result of inaccuracy, perhaps suggesting they are unlikely to abandon the system after 
experiencing one-time or periodic inaccuracy. However, it is important to note the trade-off of this slow response. It may take longer to rebuild system trust even when the system has already been accurate. Although results suggest that inaccurate priming can increase subsequent trust, it is still unknown how long a user will tolerate such inaccuracy, and how patient users are with such a system. In this experiment users had little option to stop using the system when it is inaccurate (they were free to discontinue the experiment, but as long as they were in the experiment they had to provide a trust rating). This is not true of positioning system use in reality; people are more likely to put the system away or turn it off than suffer through successive inaccurate results. As a result, it is essential for the GUI to clearly communicate to users about the current status of the system (confidence in calculating positioning results) to potentially reduce the time necessary to rebuild system trust. As mentioned before, possible reasons for low confidence should be explained to keep users engaged when the system is less accurate. Meanwhile, it is also crucial to notify users when the system is highly confident (e.g. sufficient signals for calculation or less noise in the environment). This communication to some degree reduces users' hesitation and their tendency towards abandoning the system.

\subsection{Individual Differences}

When examining trust ratings from individual users, it is not surprising to find they have different opinions from one another even at the same location. For example, there are users who argue that "very high" should be given to a calculated position that is one meter away from the actual location. However, others may be simply satisfied if the positioning error is less than five meters. Wider divergences are observed when it 
comes to inaccuracy. Some participants pointed out that calculated positions presented inside a building's outline is the baseline for an indoor positioning system. Conversely, there are beliefs that WRONG BUILDING locations can still communicate clearly if they are close enough to the actual location. Therefore, to some degree, there is not a single result which is absolutely accurate or inaccurate to all users. In addition to the diverse opinions of accuracy, users' spatial ability, familiarity with the environment and understanding of the positioning technology should be taken into account in user studies as well, as each of them plays a distinct role in users' trust evaluations. As results suggest, participants who have high spatial ability and familiarity with the environment are more sensitive to the inaccurate positioning results, which in turn results in a lower trust in the system overall. As mentioned in the literature, this overconfidence might result in distrust in spite of the system being accurate (Kantowitz, et al., 1997). On the contrary, low spatial ability and familiarity could cause overreliance in the system, which means users still choose to trust an inaccurate system because of their inability to identify the inaccuracy. However, users may find they are much more lost than they expect. Consequently, in such situations the system should notify users its confidence regarding each calculated result; such information encourages users to consider both their own knowledge and the system's ability in the real time. Interestingly, knowledge of GPS calculation principle increases trust in WRONG BUILDING locations. However, the system should not expect every user to have this kind of knowledge but take into account the overall situation of all users. 


\section{V \\ CONCULUSION}

As an alternative solution for navigating indoor environments where GPS does not function, indoor positioning systems are designed to improve the efficiency of human wayfinding through the provision of accurate and reliable location information. However, these systems are far from ubiquitously accurate and inaccuracy cannot be easily predicted by the designer. These issues increase the need to understand users' opinions of the system in relation to trust and maintaining user trust under conditions of varying accuracy. A possible response to such situations is to adjust the system appropriately during periods of unexpected but identifiable inaccuracy based on user studies. Trust has been explored widely in the use of automation; however, it has not received enough attention in the field of positioning. The existing literature evaluates user trust of a positioning system only based on one-time or average positioning accuracy and does not take into account the dynamic interaction among the user, device/system, and trust. Therefore, it is essential to understand how users interact with changes in accuracy in order to achieve more usable positioning system design. To address these concerns, an experiment was designed to examine how users trust change with the system performance from time to time. Results from this experiment highlight the complexity of trust change during the positioning processes with consideration of individual location accuracy, previous location accuracy, overall system design, as well as individual differences. Results indicate that better accuracy contributes to higher trust. However, as a result of individual differences this increase is non-linear. Interestingly, users' trust of positioning results changes with the system performance over time, which is affected by their previous experience and current accuracy. As a 
result, inaccurate priming has a positive impact on users' trust of positioning results that follow. That is to say, users' trust increases as the system performance exceeds expectations established during priming. In addition, system trust is less variable and developed more slowly than position trust. This observation emphasizes the point that the system trust is not simply shaped by a single or periodic assessment of accuracy, but maintained by consistent positioning accuracy, a comprehensible and clear interface, and appropriate basemap information.

These results provide an initial framework to understand the relationship between positioning accuracy and human trust, which further supports understanding the complex nature of trust and geospatial data, processes, and representations. Both designers of positioning systems and researchers alike can derive benefits from this research to improve the efficiency of user-system interaction during periods of suspect accuracy. As inaccuracy can occur indoors and outdoors and not only at already known locations, it is important to maintain user trust, especially when the positioning information is less reliable. This research reveals the opportunities and challenges in terms of maintaining user trust during use of positioning systems. Most importantly, the system should be consistently accurate. In the case of unexpected inaccuracy, the system's GUI should clearly communicate the reasons for decreased accuracy and system confidence as a means to decelerate trust loss and accelerate the process of rebuilding trust. In addition to reducing the negative impact of inaccuracy, this communication is also beneficial for users with different knowledge and preferences, as it helps them more clearly understand the system's current status and accuracy. From the perspective of user studies in positioning systems, it is necessary to consider individual differences when designing the experiment in order to generalize common 
interactive approaches which meet most users' satisfaction. Although the objectives of this study were successfully fulfilled, this research is not without challenges. As users' tolerance of inaccuracy is still unknown, future work should take into account the individual differences in the judgment of inaccuracy in order to develop increasing knowledge in the user system trust relationship. Besides, attention should be drawn to other scenarios with regard to the occurrence of inaccuracy, as continuous inaccuracy could occur not only in the beginning but also in the middle of the positioning process; for instance, it would be interesting to compare users' trust before and after consecutive experiences with low accuracy. 


\section{REFERENCES}

Antifakos, S., Kern, N., Schiele, B., \& Schwaninger, A. (2005). Towards improving trust in context-aware systems by displaying system confidence. Paper presented at the 7 th international conference on Human computer interaction with mobile devices \& services, University of Salzburg, Austria.

Artz, D., \& Gil, Y. (2007). A survey of trust in computer science and the Semantic Web. Web Semantics: Science, Services and Agents on the World Wide Web, 5(2), 58-71.

Beal, J. R. (2003). Contextual geolocation, a specialized application for improving indoor location awareness in wireless local area networks. Paper presented at the 36th Annual Midwest Instruction and Computing Symposium, St. Louis, Missouri.

Bell, S., Jung, W. R., \& Krishnakumar, V. (2010). Wifi-based enhanced positioning systems: Accuracy through Mapping, Calibration, and Classification. Paper presented at the 2nd ACM SIGSPATIAL International Workshop on Indoor Spatial Awareness, San Jose, CA, USA.

Bell, S., Wei, T., Jung, W. R., \& Scott, A. (2011). A conceptual model of trust for indoor positioning systems. Paper presented at the 3 rd ACM SIGSPATIAL International Workshop on Indoor Spatial Awareness, Chicago, IL, USA.

Bhasker, E. S., Brown, S. W., \& Griswold, W. G. (2004). Employing User Feedback for Fast, Accurate, Low-Maintenance Geolocationing. Paper presented at the Second IEEE Annual Conference on Pervasive Computing and Communications (PerCom 2004), Orlando, FL, USA.

Bornträger, C., Cheverst, K., Davies, N., Dix, A., Friday, A., \& Seitz, J. (2003). Experiments with multi-modal interfaces in a context-aware city guide. HumanComputer Interaction with Mobile Devices and Services, 116-130.

Chincholle, D., Goldstein, M., Nyberg, M., \& Eriksson, M. (2002). Lost or found? A usability evaluation of a mobile navigation and location-based service. Human Computer Interaction with Mobile Devices, 211-224.

Ciavarella, C., \& Paternò, F. (2004). The design of a handheld, location-aware guide for indoor environments. Personal and Ubiquitous Computing, 8(2), 82-91.

Deitrick, S., \& Edsall, R. (2009). Making Uncertainty Usable: Approaches for Visualizing Uncertainty Information. In M. Dodge, M. McDerby \& M. Turner (Eds.), Geographic Visualization: Concepts, Tools \& Applications (pp. 277-288). Chichester, UK: John Wiley \& Sons, Ltd. 
Dey, A. K. (2001). Understanding and using context. Personal and Ubiquitous Computing, 5(1), 4-7.

Dillemuth, J. (2005). Map design evaluation for mobile display. Cartography and Geographic Information Science, 32(4), 285-301.

Dillemuth, J., Goldsberry, K., \& Clarke, K. C. (2007). Choosing the scale and extent of maps for navigation with mobile computing systems. Journal of Location Based Services, 1(1), 46-61.

Flavián, C., Guinalíu, M., \& Gurrea, R. (2006). The role played by perceived usability, satisfaction and consumer trust on website loyalty. Information \& Management, 43(1), $1-14$.

Fogg, B. J., \& Tseng, H. (1999). The elements of computer credibility. Paper presented at the Proceedings of the SIGCHI conference on Human factors in computing systems: the CHI is the limit, Pittsburgh, PA, USA.

Georgy, J., Noureldin, A., Korenberg, M. J., \& Bayoumi, M. M. (2010a). Low-Cost Three-Dimensional Navigation Solution for RISS/GPS Integration Using Mixture Particle Filter. IEEE Transactions on Vehicular Technology, 59(2), 599-615.

Georgy, J., Noureldin, A., Korenberg, M. J., \& Bayoumi, M. M. (2010b). Modeling the Stochastic Drift of a MEMS-Based Gyroscope in Gyro/Odometer/GPS Integrated Navigation. IEEE Transactions on Intelligent Transportation Systems, 11(4), 856-872.

Gezici, S. (2008). A survey on wireless position estimation. Wireless Personal Communications, 44(3), 263-282.

Gorlenko, L., \& Merrick, R. (2003). No wires attached: Usability challenges in the connected mobile world. IBM Systems Journal, 42(4), 639-651.

Hazas, M., Scott, J., \& Krumm, J. (2004). Location-aware Computing Comes of Age. Computer, 37(2), 95-97.

Hegarty, M., Richardson, A. E., Montello, D. R., Lovelace, K., \& Subbiah, I. (2002). Development of a self-report measure of environmental spatial ability. Intelligence, $30(5), 425-447$.

Hightower, J. (2003). From position to place. Paper presented at the Workshop on Location-Aware Computing, Seattle, WA, USA.

Hightower, J., \& Borriello, G. (2001). A survey and taxonomy of location systems for ubiquitous computing. IEEE Computer, 34(8), 57-66. 
Irwin, S., \& Marion, E. (1992). Sex differences in spatial abilities: Evolutionary theory and data. In J. H. Barkow, L. Cosmides \& J. Tooby (Eds.), The adapted mind:

Evolutionary psychology and the generation of culture (pp. 533-549). New York, NY, US: Oxford University Press.

Ishikawa, T., Fujiwara, H., Imai, O., \& Okabe, A. (2008). Wayfinding with a GPS-based mobile navigation system: A comparison with maps and direct experience. Journal of Environmental Psychology, 28(1), 74-82.

Jonsson, I.-M., Harris, H., \& Nass, C. (2008). How accurate must an in-car information system be?: consequences of accurate and inaccurate information in cars. Paper presented at the 26th annual SIGCHI conference on Human factors in computing systems, Florence, Italy.

Jonsson, I.-M., Harris, H., Nass, C., \& Takayama, L. (2005). Got Info? Examining the Consequences of Inaccurate Information Systems. Paper presented at the 3 rd International Driving Symposium on Human Factors in Driver Assessment, Training and Vehicle Design, Rockport, MA, USA.

Kantowitz, B. H., Hanowski, R. H., \& Kantowitz, S. C. (1997). Driver reliability requirements for traffic advisory information. Ergonomics and safety of intelligent driver interfaces, 1-22.

Lee, J. D., \& See, K. A. (2002). Trust in computer technology and the implications for design and evaluation. Etiquette for Human-Computer Work: Technical Report FS-O2o2, 20-25.

Lee, J. D., \& See, K. A. (2004). Trust in automation: Designing for appropriate reliance. Human Factors: The Journal of the Human Factors and Ergonomics Society, 46(1), 50.

Li, K.-J. (2008). Indoor Space: A New Notion of Space. Paper presented at the Proceedings of the 8th International Symposium on Web and Wireless Geographical Information Systems (W2GIS 2008), Shanghai, China.

Liu, H., Darabi, H., Banerjee, P., \& Liu, J. (2007). Survey of wireless indoor positioning techniques and systems. IEEE Transactions on Systems, Man, and Cybernetics, Part C: Applications and Reviews, 37(6), 1067-1080.

Olsen, D. (2009). Building Interactive Systems: Principles for Human-Computer Interaction. Boston: Course Technology Press.

Perkins, L., Miller, J. E., Hashemi, A., \& Burns, G. (2010). Designing for HumanCentered Systems: Situational Risk as a Factor of Trust in Automation. Human Factors: The Journal of the Human Factors and Ergonomics Society, 54(25), 2130-2134. 
Roy, M. C., Dewit, O., \& Aubert, B. A. (2001). The impact of interface usability on trust in web retailers. Internet Research, 11(5), 388-398.

Sanchez, J., Rogers, W. A., Fisk, A. D., \& Rovira, E. (2011). Understanding reliance on automation: effects of error type, error distribution, age and experience. Theoretical Issues in Ergonomics Science, 1-27.

Sayda, F. (2005). Involving LBS users in data acquisition and update. Paper presented at the 8th AGILE (the Association of Geographic Information Laboratories for Europe) Conference on GIScience, Estoril Congress Center, Estoril, Portugal.

Schröter, D., Beetz, M., \& Gutmann, J.-S. (2002). Rg mapping: Learning compact and structured 2d line maps of indoor environments. Paper presented at the 11th IEEE International Workshop onRobot and Human Interactive Communication, Berlin, Germany.

Shoeb, M., Ahmad, F., \& Amin, M. (2006). Indoor wireless source localization based on area constraints. Paper presented at the SPIE Symposium on Defense and Security, Wireless Sensing and Processing Conference, Orlando, FL, USA.

Stanton, N. S., Ragsdale, S. A., \& Bustamante, E. A. (2009). The effects of system technology and probability type on trust, compliance, and reliance. Paper presented at the Human Factors and Ergonomics Society Annual Meeting, San Antonio, TX, USA.

Thirunarayan, K., Anantharam, P., Henson, C. A., \& Sheth, A. P. (2010, 17-21 May 2010). Some trust issues in social networks and sensor networks. Paper presented at the International Symposium on Collaborative Technologies and Systems (CTS), Chicago, IL, USA.

Thorndyke, P. W., \& Hayes-Roth, B. (1982). Differences in spatial knowledge acquired from maps and navigation. Cognitive psychology, 14(4), 560-589.

Tseng, S., \& Fogg, B. J. (1999). Credibility and computing technology. Communications of the ACM, 42(5), 39-44.

Verdine, B. N. (2011). Navigation experience in video game environments: Effects on spatial ability and map use skills. Vanderbilt University, Nashville, Tennessee.

Vries, P. d., Midden, C., \& Bouwhuis, D. (2003). The effects of errors on system trust, self-confidence, and the allocation of control in route planning. International Journal of Human-Computer Studies, 58(6), 719-735.

Wagealla, W., Terzis, S., \& English, C. (2003). Trust-based model for privacy control in context aware systems. Paper presented at the Second Workshop on Security in 
Ubiquitous Computing at the Fifth Annual Conference on Ubiquitous Computing (UbiComp2003), Washington, DC, USA.

Wei, E., \& Chan, A. (2007). Towards Context-Awareness in Ubiquitous Computing. Embedded and Ubiquitous Computing, 4808, 706-717. doi: 10.1007/978-3-540-770923_61

Wing, M. G., Eklund, A., \& Kellogg, L. D. (2005). Consumer-grade global positioning system (GPS) accuracy and reliability. Journal of Forestry, 103(4), 169-173.

Xiang, Z., Song, S., Chen, J., Wang, H., Huang, J., \& Gao, X. (2004). A wireless LANbased indoor positioning technology. IBM Journal of Research and Development, 48(5.6), 617-626.

Zandbergen, P. A. (2009). Accuracy of iPhone Locations: A Comparison of Assisted GPS, WiFi and Cellular Positioning. Transactions in GIS, 13, 5-25.

Zàruba, G. V., Huber, M., Kamangar, F. A., \& Chlamtac, I. (2007). Indoor location tracking using RSSI readings from a single Wi-Fi access point. Wireless networks, 13(2), 221-235. 


\section{APPENDIXIES}

I: Visiting locations (actual locations where participants stand) and result locations in all three buildings

a. Kirk Hall

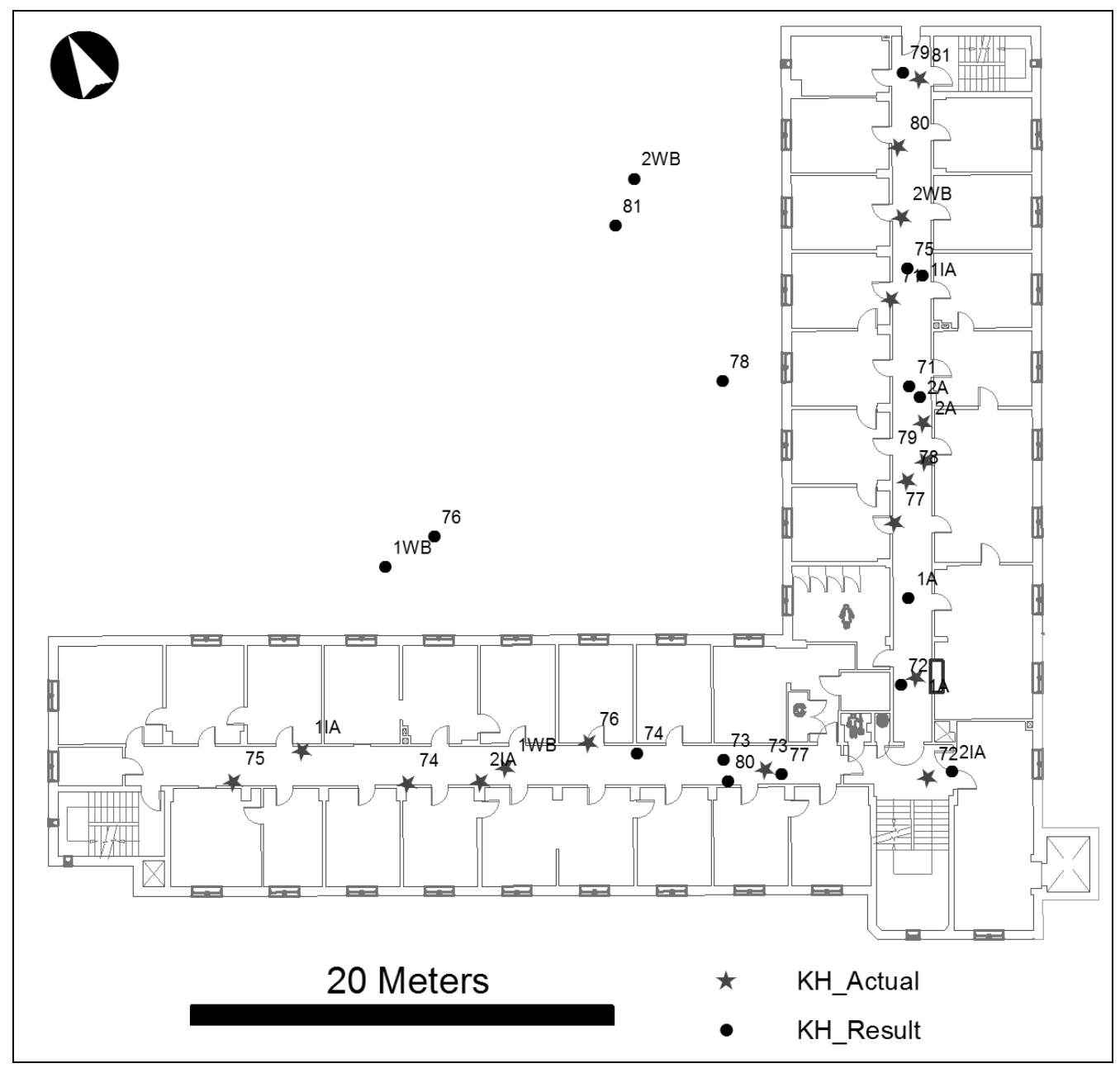


b. Agriculture Building

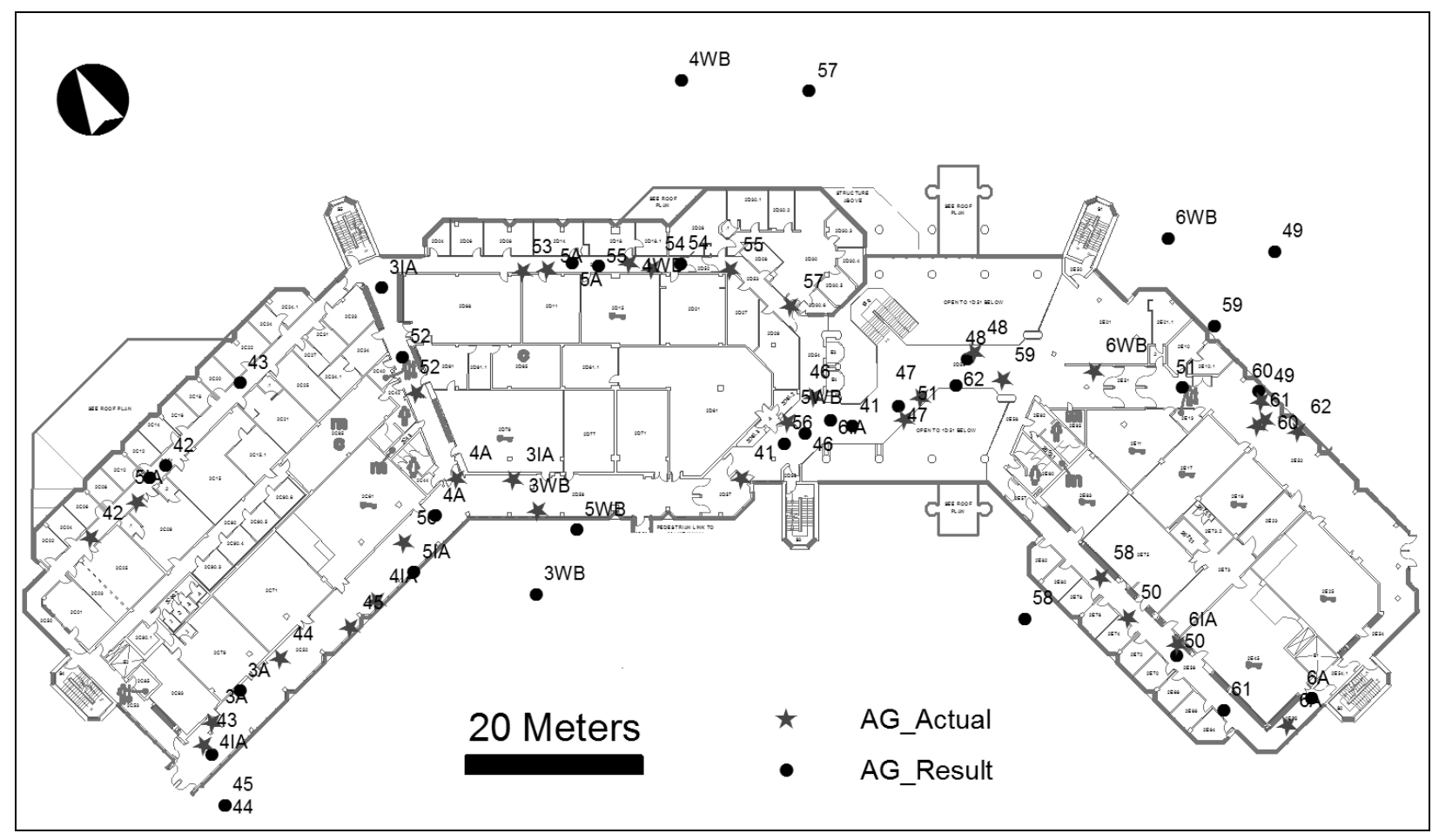

c. Engineering Building 


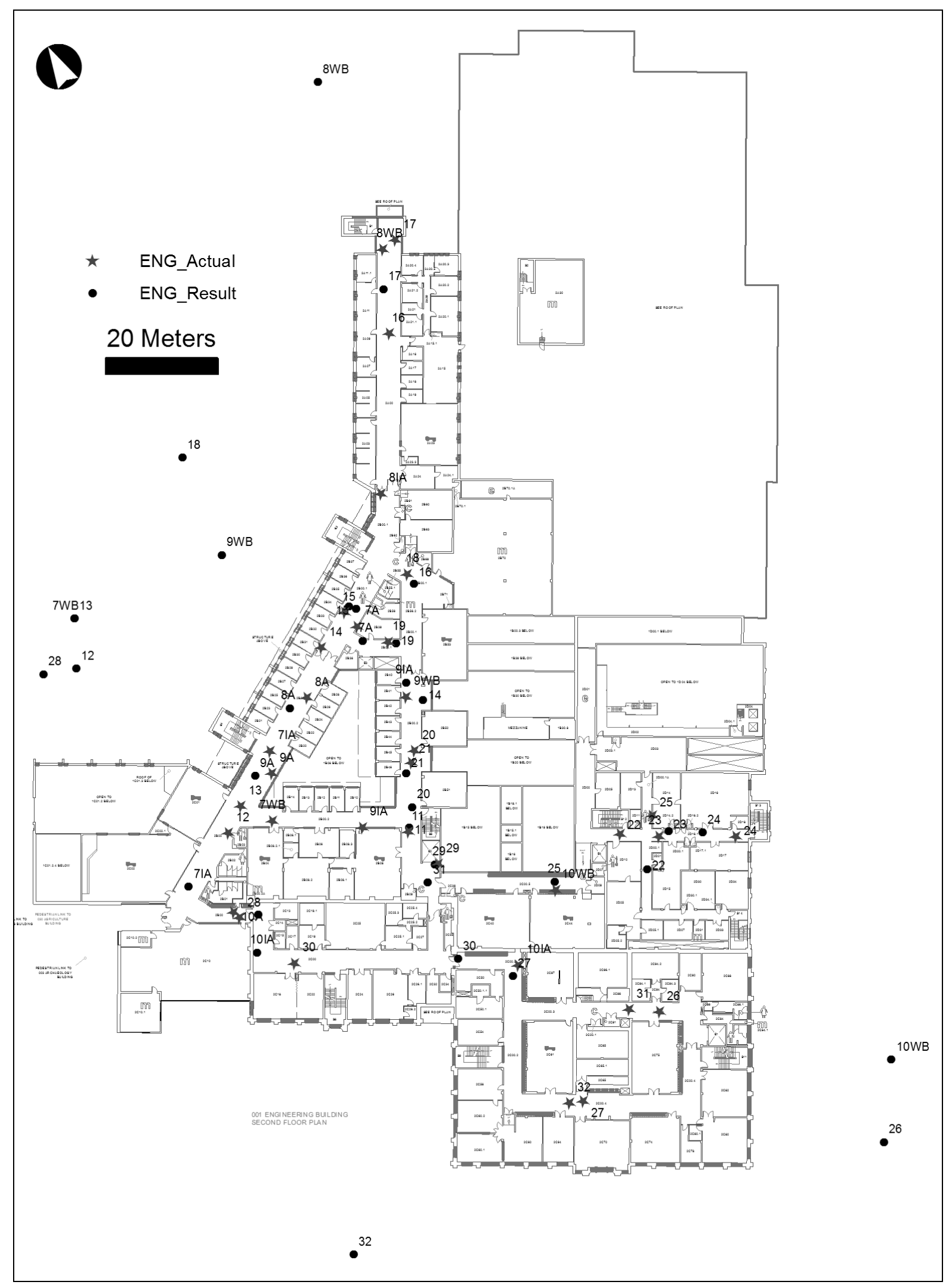


II: Attribute information of each experimental location in all three buildings

a. Priming locations

\begin{tabular}{|c|c|c|c|c|}
\hline Group & Point_ID & Building & Category & Error(m) \\
\hline \multirow{10}{*}{ Group 1} & $1 \mathrm{~A}$ & KH & A & 3.8 \\
\hline & $2 \mathrm{~A}$ & $\mathrm{KH}$ & A & 1.3 \\
\hline & $3 \mathrm{~A}$ & $\mathrm{AG}$ & A & 4.9 \\
\hline & $4 \mathrm{~A}$ & AG & A & 4.8 \\
\hline & $5 \mathrm{~A}$ & AG & A & 3.0 \\
\hline & $6 \mathrm{~A}$ & AG & A & 4.3 \\
\hline & $7 \mathrm{~A}$ & ENG & $\mathrm{A}$ & 2.7 \\
\hline & $8 \mathrm{~A}$ & ENG & A & 3.5 \\
\hline & $9 \mathrm{~A}$ & ENG & A & 2.9 \\
\hline & $10 \mathrm{~A}$ & ENG & A & 4.6 \\
\hline \multirow{10}{*}{ Group 2} & 1IA & $\mathrm{KH}$ & IA & 37.1 \\
\hline & 2 IA & $\mathrm{KH}$ & IA & 22.4 \\
\hline & 3 IA & AG & IA & 26.6 \\
\hline & 4 IA & $\mathrm{AG}$ & IA & 25.5 \\
\hline & 5 IA & $\mathrm{AG}$ & IA & 32.5 \\
\hline & $6 \mathrm{IA}$ & $\mathrm{AG}$ & IA & 46.9 \\
\hline & 7 IA & ENG & IA & 28.3 \\
\hline & $8 \mathrm{IA}$ & ENG & IA & 21.0 \\
\hline & 9 IA & ENG & IA & $27 \cdot 3$ \\
\hline & $10 \mathrm{IA}$ & ENG & IA & 46.9 \\
\hline \multirow{10}{*}{ Group 3} & $1 \mathrm{WB}$ & $\mathrm{KH}$ & WB & -- \\
\hline & $2 \mathrm{WB}$ & $\mathrm{KH}$ & WB & -- \\
\hline & $3 \mathrm{WB}$ & $\mathrm{AG}$ & WB & -- \\
\hline & $4 \mathrm{WB}$ & AG & WB & -- \\
\hline & $5 \mathrm{WB}$ & $\mathrm{AG}$ & WB & -- \\
\hline & $6 \mathrm{WB}$ & $\mathrm{AG}$ & WB & -- \\
\hline & $7 \mathrm{WB}$ & ENG & WB & -- \\
\hline & $8 \mathrm{WB}$ & ENG & WB & -- \\
\hline & $9 \mathrm{WB}$ & ENG & WB & -- \\
\hline & $10 \mathrm{WB}$ & ENG & WB & -- \\
\hline
\end{tabular}

b. Post-priming locations

\begin{tabular}{|c|c|c|c|}
\hline Point_ID & Building & Category & Error(m) \\
\hline 11 & ENG & A & 0.9 \\
\hline 12 & ENG & WB & -- \\
\hline 13 & ENG & WB & -- \\
\hline
\end{tabular}




\begin{tabular}{|c|c|c|c|}
\hline 14 & ENG & IA & 20.6 \\
\hline 15 & ENG & $\mathrm{A}$ & 2.3 \\
\hline 16 & ENG & IA & 45.1 \\
\hline 17 & ENG & $\mathrm{CON}$ & 9.0 \\
\hline 18 & ENG & WB & -- \\
\hline 19 & ENG & A & 1.5 \\
\hline 20 & ENG & $\mathrm{CON}$ & 10.0 \\
\hline 21 & ENG & A & 2.0 \\
\hline 22 & ENG & $\mathrm{CON}$ & 8.0 \\
\hline 23 & ENG & A & 2.1 \\
\hline 24 & ENG & $\mathrm{CON}$ & 6.0 \\
\hline 25 & ENG & IA & 21.0 \\
\hline 26 & ENG & WB & -- \\
\hline 27 & ENG & IA & 25.9 \\
\hline 28 & ENG & WB & -- \\
\hline 29 & ENG & A & 0.5 \\
\hline 30 & ENG & IA & 29.4 \\
\hline 31 & ENG & IA & 42.7 \\
\hline 32 & ENG & WB & -- \\
\hline 41 & $\mathrm{AG}$ & $\mathrm{CON}$ & 14.0 \\
\hline 42 & $\mathrm{AG}$ & $\mathrm{CON}$ & 12.0 \\
\hline 43 & AG & IA & 41.6 \\
\hline 44 & $\mathrm{AG}$ & WB & -- \\
\hline 45 & $\mathrm{AG}$ & WB & -- \\
\hline 46 & AG & $\mathrm{A}$ & 4.2 \\
\hline 47 & $\mathrm{AG}$ & A & 2.6 \\
\hline 48 & AG & $\mathrm{A}$ & 1.0 \\
\hline 49 & AG & WB & -- \\
\hline 50 & $\mathrm{AG}$ & $\mathrm{CON}$ & 7.0 \\
\hline 51 & $\mathrm{AG}$ & IA & 31.8 \\
\hline 52 & AG & A & 4.4 \\
\hline 53 & AG & IA & 48.3 \\
\hline 54 & AG & $\mathrm{A}$ & 3.4 \\
\hline 55 & AG & $\mathrm{CON}$ & 15.0 \\
\hline 56 & $\mathrm{AG}$ & IA & 44.8 \\
\hline 57 & $\mathrm{AG}$ & WB & -- \\
\hline 58 & $\mathrm{AG}$ & WB & -- \\
\hline 59 & $\mathrm{AG}$ & WB & -- \\
\hline 60 & $\mathrm{AG}$ & $\mathrm{A}$ & 3.4 \\
\hline 61 & AG & IA & 32.5 \\
\hline 62 & AG & IA & 39.2 \\
\hline
\end{tabular}




\begin{tabular}{|c|c|c|c|}
\hline 71 & $\mathrm{KH}$ & $\mathrm{A}$ & 4.2 \\
\hline 72 & $\mathrm{KH}$ & $\mathrm{A}$ & 4.6 \\
\hline 73 & $\mathrm{KH}$ & $\mathrm{A}$ & 2.0 \\
\hline 74 & $\mathrm{KH}$ & $\mathrm{CON}$ & 11.0 \\
\hline 75 & $\mathrm{KH}$ & $\mathrm{IA}$ & 40.2 \\
\hline 76 & $\mathrm{KH}$ & WB & -- \\
\hline 77 & $\mathrm{KH}$ & $\mathrm{CON}$ & 13.0 \\
\hline 78 & $\mathrm{KH}$ & WB & -- \\
\hline 79 & $\mathrm{KH}$ & IA & 18.5 \\
\hline 80 & $\mathrm{KH}$ & IA & 31.1 \\
\hline 81 & $\mathrm{KH}$ & WB & -- \\
\hline
\end{tabular}

III: Building familiarity and GPS experience Survey

Participant ID: Age:

Gender: M F

1. How familiar are you with the structure of Kirk Hall in general? Please choose a number to rate your familiarity ( $1=$ not at all familiar, $7=$ very familiar).

$\begin{array}{lllllll}1 & 2 & 3 & 4 & 5 & 6 & 7\end{array}$

2. How familiar are you with the structure of Agriculture Building in general? Please choose a number to rate your familiarity ( $1=$ not at all familiar, $7=$ very familiar).

$\begin{array}{lllllll}1 & 2 & 3 & 4 & 5 & 6 & 7\end{array}$

3. How familiar are you with the structure of Engineering Building in general? Please choose a number to rate your familiarity ( $1=$ not at all familiar, $7=$ very familiar).

$\begin{array}{lllllll}1 & 2 & 3 & 4 & 5 & 6 & 7\end{array}$

4. Have you ever used a positioning system to find locations (like GPS, etc.)?
A. Yes
B. No

If yes, please indicate how often have you used such a system (1=lowest frequency, $7=$ highest frequency).

$\begin{array}{lllllll}1 & 2 & 3 & 4 & 5 & 6 & 7\end{array}$ 
5. How much do you know about the basic principle of positioning systems? (1= Nothing, $7=$ A lot $)$.

$\begin{array}{lllllll}1 & 2 & 3 & 4 & 5 & 6 & 7\end{array}$ 